\title{
Observer-Based Controllers for Incrementally Quadratic Nonlinear Systems with Disturbances
}

\author{
Xiangru Xu, Behçet Açıkmeşe, Martin J. Corless
}

\begin{abstract}
Robust global stabilization of nonlinear systems by observer-based feedback controllers is a challenging task. This paper investigates the problem of designing observer-based stabilizing controllers for incrementally quadratic nonlinear systems with external disturbances. The nonlinearities considered in the system model satisfy the incremental quadratic constraints, which are characterized by incremental multiplier matrices and encompass many common nonlinearities. The simultaneous search for the observer and the controller gain matrices is formulated as a feasibility problem of linear matrix inequalities, for two parameterizations (i.e., the block diagonal parameterization and the block anti-triangular parameterization) of the incremental multiplier matrices, respectively. The closed-loop system implementing the observer-based feedback controller is proven to be input-to-state stable with respect to external disturbances. Using the proposed continuous-time observer-based controllers, eventtriggered controllers with time regularization are constructed for globally Lipschitz systems, such that the closed-loop system is Zeno-free and input-to-state practically stable.
\end{abstract}

\section{INTRODUCTION}

As the state variables of a system are difficult or expensive to measure in practice, output feedback control design has received a lot of attention (see, e.g., [1], [2], [3], [4]) and found applications in biological systems [5], [6], [7, mechanical systems [8], [9], [10], power systems [11], [12], and networked control systems [13], [14], among others. For linear systems, the output feedback stabilizing control design problem can be solved by designing the state-feedback controller and the state observer independently, which is known as the controller-observer separation principle. For nonlinear systems, a certainty-equivalence implementation of a globally stabilizing state-feedback controller with an asymptotic observer can lead to finite escape time (e.g., see the counter-examples in [15], [16]), which makes observer-based stabilizing controller design a challenging problem [17]. By using a high-gain observer [18], [19], separation principles for input-output linearizable systems were studied in [20], [21], [22], [23] and semiglobal asymptotic stability of the resulting closed-loop systems was proven in these papers. Separation principles for some other special class of nonlinear systems were also investigated, such as bilinear systems [24], [25], non-affine nonlinear systems [26], systems with nondecreasing or slope-restricted nonlinearities [27], [28], [29],

$\mathrm{X} . \mathrm{Xu}$ is with the Department of Mechanical Engineering, University of Wisconsin-Madison, Madison, WI, USA (email: xiangru. xu@wisc.edu).

B. Açımeşe is with the Department of Aeronautics \& Astronautics, University of Washington, Seattle, WA, USA (email: behcet @uw. edu).

M. J. Corless is with the School of Aeronautics \& Astronautics, Purdue University, W. Lafayette, IN, USA (email: corless@purdue. edu). and cascaded systems [30], [31]. Apart from the certaintyequivalence approach, interdependent design of the controller and the observer was investigated in [32], [33], [34].

Linear matrix inequalities (LMIs) provide a computationally efficient approach for the synthesis of observer-based output feedback controllers [35], where the main difficulty lies in the coupling between the unknown matrices of the observer and the controller and the Lyapunov matrices. For linear systems, LMI-based conditions were proposed for the robust observerbased stabilization of linear systems with state perturbations [36] or with parametric uncertainties [37], [38]. For nonlinear systems, the synthesis problem is often formulated as the feasibility of bilinear matrix inequalities (BMIs), which is known to be an NP-hard problem [39]. Different approaches that aim to transform the non-convex BMI conditions to convex LMI conditions have been proposed: [38] studied observerbased controller design for Lipschitz nonlinear systems with uncertain parameters, and developed an LMI-based design technique that relies on the linearization of the corresponding BMIs; [40] investigated observer-based control design for the interconnection of a linear system and an uncertain nonlinear operator satisfying the integral quadratic constraint, and proposed a sequential LMI algorithm to solve BMIs; [41] studied output feedback control of discrete-time parametric uncertain Lure systems, and developed an LMI-based iterative algorithm to solve BMIs. Moreover, [42] investigated $H_{\infty}$ stabilization of discrete-time globally Lipschitz nonlinear systems, and provided LMI-based conditions that compute simultaneously the observer and controller gains; [43] considered asymptotic stabilization of continuous-time Lipschitz nonlinear systems and developed LMI-based conditions that synthesize the gain matrices of the observer-based controller.

This paper considers observer-based output feedback global stabilization of a class of nonlinear systems whose nonlinearities satisfy incremental quadratic constraints. The incremental quadratic constraint is characterized by an incremental quadratic inequality with incremental multiplier matrices [44], [45], [46], [47], [48], [49]. This characterization with incremental multiplier matrices provides a general framework to represent many common classes of nonlinearities (e.g., globally Lipschitz nonlinearities, incrementally sector bounded nonlinearities, non-decreasing nonlinearities and the polytopic Jacobian nonlinearities), implying a wide range of applicability for the proposed theoretical results. Observer design for systems with nonlinearities satisfying incremental quadratic constraints was studied in [48], which was later generalized to the systems with bounded exogenous disturbances in [50]. Observer-based control design for some special classes of 
incrementally quadratic nonlinear systems have been investigated in [28], [43], [51], [52], [53].

Motivated by the development of networked control systems, event-triggered control (ETC) has recently received a lot of attention as it provides a new control paradigm to reduce the resource consumption of networked control systems whose communication bandwidth and computational power are usually limited [54], [55], [56]. Most of the ETC results assume that full-state information is available, but this assumption is restrictive since many systems only have information on their measured outputs. Extending results on observer-based, event-triggered control design from linear systems (e.g., see [57], [58], [59]) to nonlinear systems is difficult [60]. Existing results on ETC design for nonlinear systems mostly assume that the continuous-time observer-based controllers are already given, but the observers and controllers themselves can be hard to construct. When external disturbances or measurement noise are present, the triggering rules also need to be carefully designed to rule out the Zeno phenomenon [60], [61], [62], e.g., using time regularization to enforce a built-in lower bound for inter-execution times.

The main contributions of the paper are summarized as follows. For incrementally quadratic nonlinear systems affected by external disturbances and measurement noise, LMIbased sufficient conditions are developed for the design of robust stabilizing observer-based controllers. The simultaneous search for the observer and the controller gain matrices is formulated as a feasibility problem of LMIs when the incremental multiplier matrices are parameterized as the block diagonal matrices or the block anti-triangular matrices. The resulting closed-loop system is proven to be input-to-state stable with respect to disturbances. Using the proposed continuous-time observer-based controller, event-triggered controllers are constructed for globally Lipschitz systems affected by external disturbances and measurement noise where the triggering rule is designed with an enforced positive lower-bound on interexecution times. The resulting closed-loop system is Zeno-free and input-to-state practically stable with respect to external disturbances. A preliminary version of this work appeared in [63]. The present paper is different from [63] in the following important ways: the system model considered is subject to external disturbances and measurement noise; the event-triggered mechanism is considered; complete proofs are included, and more discussion is added. The remainder of the paper is organized as follows: Section $\mathrm{II}$ introduces preliminaries on incremental quadratic constraints and input-to-state practical stability, Section III develops LMI-based conditions for the design of robust stabilizing observer-based controllers for two parameterizations of the incremental multiplier matrices, Section IV] presents the event-triggered controller design, Section $\mathrm{V}$ provides a simulation example, and Section VI provides the conclusions.

Notation. $\mathbb{R}_{0}^{+}$denotes the set of non-negative real numbers; $\|x\|$ denotes the 2 -norm of a vector $x ;\|P\|$ denotes the maximum singular value of a matrix $P ; \lambda_{m}(P)$ and $\lambda_{M}(P)$ denote the minimum and maximum eigenvalues of a symmmetric matrix $P$, respectively; $I_{n}$ denotes an identity matrix of size $n ; \mathbf{0}_{n_{1} \times n_{2}}$ and $\mathbf{0}_{n}$ denote the zero matrix of size $n_{1} \times n_{2}$ and the zero vector of size $n$, respectively, where the subscript will be omitted when clear from context. For symmetric matrices, * denotes entries whose values follow from symmetry. For a matrix $M, M \succ 0, M \succeq 0, M \prec 0, M \preceq 0$ mean $M$ is positive definite, positive semi-definite, negative definite, and negative semi-definite, respectively. A continuous function $f: \mathbb{R}_{0}^{+} \rightarrow \mathbb{R}_{0}^{+}$belongs to class $\mathcal{K}$ (denoted as $f \in \mathcal{K}$ ) if it is strictly increasing and $f(0)=0 ; f$ belongs to class $\mathcal{K}_{\infty}$ (denoted as $\left.f \in \mathcal{K}_{\infty}\right)$ if $f \in \mathcal{K}$ and $f(r) \rightarrow \infty$ as $r \rightarrow \infty$. A continuous function $f: \mathbb{R}_{0}^{+} \times \mathbb{R}_{0}^{+} \rightarrow \mathbb{R}_{0}^{+}$belongs to class $\mathcal{K} \mathcal{L}$ (denoted as $f \in \mathcal{K} \mathcal{L}$ ) if for each fixed $s$, the function $f(\cdot, s) \in \mathcal{K}_{\infty}$ and for each each fixed $r$, the function $f(r, \cdot)$ is decreasing and $f(r, s) \rightarrow 0$ as $s \rightarrow 0$.

\section{PRELIMINARIES}

Consider the following nonlinear system

$$
\left\{\begin{array}{l}
\dot{x}=A x+B u+E p(q)+E_{w} w, \\
y=C x+D u+F_{w} w, \\
q=C_{q} x,
\end{array}\right.
$$

where $x \in \mathbb{R}^{n_{x}}$ is the state, $u \in \mathbb{R}^{n_{u}}$ is the control input, $y \in \mathbb{R}^{n_{y}}$ is the measured output, $p: \mathbb{R}^{n_{q}} \rightarrow \mathbb{R}^{n_{p}}$ is the known nonlinearity of the system, $w \in \mathbb{R}^{n_{w}}$ is the unknown external disturbance or measurement noise, and $A \in \mathbb{R}^{n_{x} \times n_{x}}, B \in$ $\mathbb{R}^{n_{x} \times n_{u}}, C \in \mathbb{R}^{n_{y} \times n_{x}}, D \in \mathbb{R}^{n_{y} \times n_{u}}, C_{q} \in \mathbb{R}^{n_{q} \times n_{x}}, E \in$ $\mathbb{R}^{n_{x} \times n_{p}}, E_{w} \in \mathbb{R}^{n_{x} \times n_{w}}, F_{w} \in \mathbb{R}^{n_{y} \times n_{w}}$ are constant matrices of appropriate dimensions.

The characterization of the nonlinearity $p$ is based on incremental multiplier matrices [48], [49].

Definition 1. Given a function $p: \mathbb{R}^{n_{q}} \rightarrow \mathbb{R}^{n_{p}}$, a symmetric matrix $M \in \mathbb{R}^{\left(n_{q}+n_{p}\right) \times\left(n_{q}+n_{p}\right)}$ is called an incremental multiplier matrix $(\delta-M M)$ for $p$ if it satisfies the following incremental quadratic constraint for all $q_{1}, q_{2} \in \mathbb{R}^{n_{q}}$ :

$$
\left(\begin{array}{l}
\delta q \\
\delta p
\end{array}\right)^{\top} M\left(\begin{array}{l}
\delta q \\
\delta p
\end{array}\right) \geq 0
$$

where $\delta q=q_{2}-q_{1}, \delta p=p\left(q_{2}\right)-p\left(q_{1}\right)$.

For a given nonlinearity $p$, its $\delta$-MM is not unique. Denote $\mathcal{M}$ as the set of incremental multiplier matrices for $p$. If $M \in$ $\mathcal{M}$, then $\lambda M \in \mathcal{M}$ for any $\lambda \geq 0$.

Remark 1. The global Lipschitz condition $\left\|p\left(q_{2}\right)-p\left(q_{1}\right)\right\| \leq$ $\gamma\left\|q_{2}-q_{1}\right\|$ where $\gamma>0$ can be expressed in the form of (2) with

$$
M=\left(\begin{array}{cc}
\gamma^{2} I & \mathbf{0} \\
\mathbf{0} & -I
\end{array}\right) .
$$

The incrementally sector bounded nonlinearity $(\delta p-$ $\left.K_{1} \delta q\right)^{\top} S\left(\delta p-K_{2} \delta q\right) \leq 0$ where $S=S^{\top}$ can be expressed in the form of (2) with

$$
M=\left(\begin{array}{cc}
-K_{1}^{\top} S K_{2}-K_{2}^{\top} S K_{1} & * \\
S\left(K_{1}+K_{2}\right) & -2 S
\end{array}\right) .
$$

The nondecreasing nonlinearity, which satisfies $\delta p^{\top} \delta q \geq 0$, can be expressed in the form of (2) with

$$
M=\left(\begin{array}{ll}
\mathbf{0} & I \\
I & \mathbf{0}
\end{array}\right) \text {. }
$$


Refer to [48], [49] for some other nonlinearities that can be expressed using the incremental quadratic constraint.

Next, we introduce input-to-state practical stability and its characterization using Lyapunov functions. Consider the system

$$
\dot{x}=f(x, u)
$$

where $f: \mathbb{R}^{n_{x}} \times \mathbb{R}^{n_{u}} \rightarrow \mathbb{R}^{n_{x}}$ is a locally Lipschitz function and $u: \mathbb{R} \rightarrow \mathbb{R}^{n_{u}}$ is a measurable essentially bounded input. Define $x\left(t, x_{0}, u\right)$ as the solution of (6) with initial state $x_{0}$ and input $u$, which satisfies $x\left(0, x_{0}, u\right)=x_{0}$.

Definition 2. (Def. 2.1 of [64]) The system (6] is called inputto-state practically stable (ISpS) w.r.t. $u$, if there exist functions $\beta_{1} \in \mathcal{K} \mathcal{L}, \beta_{2} \in \mathcal{K}$ and a non-negative constant $d$ such that for every initial state $x_{0}$ and every measurable essentially bounded $u$ defined on $[0, \infty)$, the solution $x\left(t, x_{0}, u\right)$ exists on $[0, \infty)$ and satisfies

$$
\left\|x\left(t, x_{0}, u\right)\right\| \leq \beta_{1}\left(\left\|x_{0}\right\|, t\right)+\beta_{2}\left(\|u\|_{\infty}\right)+d, \forall t \geq 0
$$

where $\|u\|_{\infty}:=$ ess $\sup _{t \geq 0}\|u(t)\|$.

When (7) is satisfied with $d=0$, the system is said to be input-to-state stable (ISS) w.r.t. $u$ [65].

Definition 3. (Remark 2.2 of [64]) A smooth function $V$ : $\mathbb{R}^{n} \rightarrow \mathbb{R}$ is said to be an ISpS-Lyapunov function for the system (6) if $V$ is radially unbounded, positive definite and there exist functions $\gamma \in \mathcal{K}_{\infty}, \chi \in \mathcal{K}$ and a non-negative constant $d$ such that the following condition holds:

$$
\nabla V(x)^{T} f(x, u) \leq-\gamma(\|x\|)+\chi(\|u\|)+d .
$$

Instead of requiring inequality (8), the ISpS-Lyapunov function can be also defined equivalently as follows: a smooth, positive definite, radially unbounded function $V$ is an ISpSLyapunov function for the system (6) if there exist a positivedefinite function $\gamma$, a class $\mathcal{K}$ function $\chi$ and a non-negative constant $d$ such that the following condition holds (Def. 2.2 of [64]):

$$
\|x\| \geq \chi(\|u\|)+d \Rightarrow \nabla V(x)^{T} f(x, u) \leq-\gamma(\|x\|) .
$$

The existence of an ISpS-Lyapunov function is a necessary and sufficient condition for the ISpS property.

Proposition 1. [66] The system (6) is ISpS (resp. ISS) if and only if it has an ISpS-(resp. ISS-) Lyapunov function.

In particular, if there exist a symmetric and positive definite matrix $P=P^{\top} \succ 0$, two constants $\alpha>0, d \geq 0$ and a function $\chi \in \mathcal{K}_{\infty}$ such that the positive definite function $V(x)=x^{\top} P x$ satisfies

$$
\nabla V(x)^{T} f(x, u) \leq-\alpha V(x)+\chi(\|u\|)+d,
$$

then $V$ is an ISpS-Lyapunov function satisfying (8) with $\gamma(\|x\|)=\alpha \lambda_{m}(P)\|x\|^{2}$, implying that 6 is ISpS w.r.t. $u$.

\section{LMI-BASED CONDITIONS FOR ROBUST GLOBAL STABILIZATION OF INCREMENTALLY QUADRATIC NONLINEAR SYSTEMS}

Consider a system described by (1) where the nonlinear term $p$ satisfies the incremental quadratic constraint (2) for some $M \in \mathcal{M}$. In this section, a continuous-time observer and a feedback controller will be designed for (1), such that the closed-loop system is ISS w.r.t. $w$. LMI-based sufficient conditions will be given for the simultaneous design of the observer and controller gain matrices.

The following observer is proposed:

$$
\left\{\begin{array}{l}
\dot{\hat{x}}=A \hat{x}+B u+E p\left(\hat{q}+L_{1}(\hat{y}-y)\right)+L_{2}(\hat{y}-y), \\
\hat{y}=C \hat{x}+D u, \\
\hat{q}=C_{q} \hat{x},
\end{array}\right.
$$

where $L_{1}, L_{2}$ are gain matrices to be designed. This observer contains a copy of the plant and two correction terms, the nonlinear injection term $L_{1}(\hat{y}-y)$ and the Luenberger-type correction term $L_{2}(\hat{y}-y)$. Based on observer (11), we design the feedback controller $u$ as

$$
u=k(\hat{x})
$$

where $k: \mathbb{R}^{n_{x}} \rightarrow \mathbb{R}^{n_{u}}$ is a function that has the form of

$$
k(x)=K_{1} x+K_{2} p\left(C_{q} x\right)
$$

with gain matrices $K_{1} \in \mathbb{R}^{n_{u} \times n_{x}}, K_{2} \in \mathbb{R}^{n_{u} \times n_{p}}$ to be designed. Defining the estimation error by

$$
e(t)=x(t)-\hat{x}(t)
$$

the input (12) can be rewritten as

$$
u=k(x)-\Delta k(x, \hat{x})
$$

where $\Delta k(x, \hat{x})=k(x)-k(\hat{x})$. Recalling (13), $\Delta k$ can be expressed as $\Delta k=K_{1} e-K_{2} \Delta p$ where

$$
\Delta p=p(\hat{q})-p(q) \text {. }
$$

The closed-loop system resulting from the observer-based controller (12) can now be expressed as

$$
\left\{\begin{array}{l}
\dot{x}=\left(A+B K_{1}\right) x+\left(E+B K_{2}\right) p-B \Delta k+E_{w} w, \\
\dot{e}=\left(A+L_{2} C\right) e-E \delta p+\left(E_{w}+L_{2} F_{w}\right) w
\end{array}\right.
$$

where

$$
\left\{\begin{array}{l}
\delta p=p(q+\delta q)-p(q), \\
\delta q=-\left(C_{q}+L_{1} C\right) e-L_{1} F_{w} w .
\end{array}\right.
$$

Defining $z=\left(\begin{array}{l}x \\ e\end{array}\right)$, dynamics (15) are expressed compactly as

$$
\dot{z}=A_{c} z+H_{1} p+H_{2} \delta p+H_{3} \Delta p+H_{4} w
$$

where $\Delta p$ is given in (14), $\delta p$ is given in (16), and

$$
A_{c}=\left(\begin{array}{cc}
A+B K_{1} & -B K_{1} \\
\mathbf{0} & A+L_{2} C
\end{array}\right) \text {, }
$$




$$
\left\{\begin{array}{l}
H_{1}=\left(\begin{array}{c}
E+B K_{2} \\
\mathbf{0}
\end{array}\right), H_{2}=\left(\begin{array}{c}
\mathbf{0} \\
-E
\end{array}\right), \\
H_{3}=\left(\begin{array}{c}
B K_{2} \\
\mathbf{0}
\end{array}\right), H_{4}=\left(\begin{array}{c}
E_{w} \\
E_{w}+L_{2} F_{w}
\end{array}\right) .
\end{array}\right.
$$

The following proposition provides a sufficient condition for the closed-loop system (17) to be ISS w.r.t. $w$.

Proposition 2. Consider the system described by (1)-(2) with $p(\mathbf{0})=\mathbf{0}$. Suppose that there exist matrices $L_{1} \in$ $\mathbb{R}^{n_{q} \times n_{y}}, L_{2} \in \mathbb{R}^{n_{x} \times n_{y}}, K_{1} \in \mathbb{R}^{n_{u} \times n_{x}}, K_{2} \in \mathbb{R}^{n_{u} \times n_{p}}, P \in$ $\mathbb{R}^{2 n_{x} \times 2 n_{x}}$ with $P \succ 0$, and real numbers $\alpha_{0}>0, \mu>0, \sigma_{1} \geq$ $0, \sigma_{2} \geq 0, \sigma_{3} \geq 0$ such that

$$
\begin{aligned}
\left(\begin{array}{cc}
S_{0} & S_{1} \\
* & \mathbf{0}
\end{array}\right) & +\sigma_{1} S_{2}^{\top} M S_{2}+\sigma_{2} S_{3}^{\top} M S_{3}+\sigma_{3} S_{4}^{\top} M S_{4} \\
& -\mu S_{5}^{\top} S_{5} \preceq 0
\end{aligned}
$$

where

$$
\begin{aligned}
& S_{0}=P A_{c}+A_{c}^{\top} P+\alpha_{0} P, \\
& S_{1}=\left(\begin{array}{llll}
P H_{1} & P_{2} H_{2} & P_{3} & P H_{4}
\end{array}\right),
\end{aligned}
$$

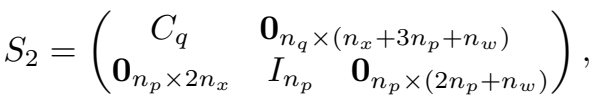

$$
\begin{aligned}
& S_{3}=\left(\begin{array}{cccc}
\mathbf{0}_{n_{q} \times n_{x}} & -\left(C_{q}+L_{1} C\right) & \mathbf{0}_{n_{q} \times 3 n_{p}} & -L_{1} F_{w} \\
\mathbf{0}_{n_{p} \times\left(2 n_{x}+n_{p}\right)} & I_{n_{p}} & \mathbf{0}_{n_{p} \times\left(n_{p}+n_{w}\right)}
\end{array}\right), \\
& S_{4}=\left(\begin{array}{ccc}
\mathbf{0}_{n_{q} \times n_{x}} & -C_{q} & \mathbf{0}_{n_{q} \times\left(3 n_{p}+n_{w}\right)} \\
\mathbf{0}_{n_{p} \times\left(2 n_{x}+2 n_{p}\right)} & I_{n_{p}} & \mathbf{0}_{n_{p} \times n_{w}}
\end{array}\right), \\
& S_{5}=\left(\begin{array}{ll}
\mathbf{0}_{n_{w} \times\left(2 n_{x}+3 n_{p}\right)} & I_{n_{w}}
\end{array}\right) .
\end{aligned}
$$

Then the closed-loop system (17) is ISS w.r.t. $w$ and satisfies $\dot{V} \leq-\alpha_{0} V+\mu\|w\|^{2}$ where $V(z)=z^{\top} P z$.

Proof. Since $M$ is a $\delta$-MM for $p$ and $p(\mathbf{0})=\mathbf{0}$, it holds that $\left(\begin{array}{l}q \\ p\end{array}\right)^{\top} M\left(\begin{array}{l}q \\ p\end{array}\right) \geq 0,\left(\begin{array}{l}\delta q \\ \delta p\end{array}\right)^{\top} M\left(\begin{array}{l}\delta q \\ \delta p\end{array}\right) \geq 0,\left(\begin{array}{c}\Delta q \\ \Delta p\end{array}\right)^{\top} M\left(\begin{array}{c}\Delta q \\ \Delta p\end{array}\right) \geq 0$, where $\delta p, \delta q$ are given in (16,,$\Delta p$ is given in (14), and

$$
\Delta q=C_{q} \hat{x}-C_{q} x=-C_{q} e .
$$

With $\xi=\left(x^{\top} e^{\top} p^{\top} \delta p^{\top} \Delta p^{\top} w^{\top}\right)^{\top}$,

$$
\left(\begin{array}{l}
q \\
p
\end{array}\right)=S_{2} \xi, \quad\left(\begin{array}{l}
\delta q \\
\delta p
\end{array}\right)=S_{3} \xi, \quad\left(\begin{array}{c}
\Delta q \\
\Delta p
\end{array}\right)=S_{4} \xi .
$$

Hence, $\xi^{\top} S_{2}^{\top} M S_{2} \xi \geq 0, \xi^{\top} S_{3}^{\top} M S_{3} \xi \geq 0, \xi^{\top} S_{4}^{\top} M S_{4} \xi \geq 0$. Pre- and post-multiply 20 by $\xi^{\top}$ and $\xi$, respectively. Since $\sigma_{1}, \sigma_{2}, \sigma_{3}$ are non-negative, we obtain that

$$
\xi^{\top}\left(\begin{array}{cc}
S_{0} & S_{1} \\
* & \mathbf{0}
\end{array}\right) \xi-\mu \xi^{\top} S_{5}^{\top} S_{5} \xi \leq 0 .
$$

Consider the positive definite function defined by $V(z)=$ $z^{\top} P z$. Then, it is easy to check that $\dot{V}+\alpha_{0} V-\mu w^{\top} w$ is equal to the left hand side of (22) where $\dot{V}$ is the derivative of $V$ along the trajectories of (17). Therefore, $V$ is an ISSLyapunov function since $\dot{V} \leq-\alpha_{0} V+\mu\|w\|^{2}$. The conclusion follows from Proposition 1

Clearly, matrix inequality (20) is not a LMI. In the next two subsections, we will consider two parameterizations of the
$\delta$-MM $M$ and provide LMI conditions which can be used to solve for $M$ and gain matrices $L_{1}, L_{2}, K_{1}, K_{2}$ simultaneously.

\section{A. Block Diagonal Parameterization}

This subsection considers a block diagonal parameterization of the $\delta$-MM for $p$. We first make the following two assumptions on the parameterizations of $M$.

Assumption 1. There exist a set $\mathcal{N}_{1}$ of matrix pairs $\left(X_{1}, Y_{1}\right)$ with $X_{1} \in \mathbb{R}^{n_{q} \times n_{q}}, Y_{1} \in \mathbb{R}^{n_{p} \times n_{p}}$ symmetric, and an invertible matrix $T_{1}$ with

$$
T_{1}=\left(\begin{array}{ll}
T_{11} & T_{12} \\
T_{13} & T_{14}
\end{array}\right)
$$

and $T_{14} \in \mathbb{R}^{n_{p} \times n_{p}}$ invertible, such that $M_{1}$ given below is a $\delta$-MM of $p$ for all $\left(X_{1}, Y_{1}\right) \in \mathcal{N}_{1}$ :

$$
M_{1}=T_{1}^{\top} \tilde{M}_{1} T_{1} \text { where } \tilde{M}_{1}=\left(\begin{array}{cc}
X_{1} & \mathbf{0} \\
\mathbf{0} & -Y_{1}
\end{array}\right) .
$$

Assumption 2. There exist a set $\mathcal{N}_{2}$ of matrix pairs $\left(X_{2}, Y_{2}\right)$ with $X_{2} \in \mathbb{R}^{n_{q} \times n_{q}}, Y_{2} \in \mathbb{R}^{n_{p} \times n_{p}}$ symmetric and invertible, and an invertible matrix $T_{2}$ with

$$
T_{2}=\left(\begin{array}{ll}
T_{21} & T_{22} \\
T_{23} & T_{24}
\end{array}\right)
$$

and $T_{24} \in \mathbb{R}^{n_{p} \times n_{p}}$ invertible, such that $M_{2}$ given below is a $\delta$-MM of $p$ for all $\left(X_{2}, Y_{2}\right) \in \mathcal{N}_{2}$ :

$$
M_{2}=T_{2}^{\top} \tilde{M}_{2} T_{2} \text { where } \tilde{M}_{2}=\left(\begin{array}{cc}
X_{2}^{-1} & \mathbf{0} \\
\mathbf{0} & -Y_{2}^{-1}
\end{array}\right) .
$$

Remark 2. For the globally Lipschitz nonlinearity $\| p\left(q_{2}\right)-$ $p\left(q_{1}\right)\|\leq \gamma\| q_{2}-q_{1} \|$, the matrix $M$ in (3) satisfies Assumption 1 and 2 if we choose

$$
T_{1}=T_{2}=\left(\begin{array}{cc}
\gamma I & \mathbf{0} \\
\mathbf{0} & I
\end{array}\right), \mathcal{N}_{1}=\mathcal{N}_{2}=\{(\lambda I, \lambda I) \mid \lambda>0\} .
$$

For the incrementally sector bounded nonlinearity ( $\delta p-$ $\left.K_{1} \delta q\right)^{\top} S\left(\delta p-K_{2} \delta q\right) \leq 0$ where $S$ is symmetric and invertible, the matrix $M$ in (4) satisfies Assumption 1 and 2 if we choose

$T_{1}=T_{2}=\left(\begin{array}{cc}K_{2}-K_{1} & \mathbf{0} \\ K_{2}+K_{1} & -2 I\end{array}\right), \mathcal{N}_{1}=\mathcal{N}_{2}=\{(\lambda S, \lambda S) \mid \lambda>0\}$.

For the nondecreasing nonlinearity $\delta p^{\top} \delta q \geq 0$, the matrix $M$ in (5) satisfies Assumption 17 and 2 if we choose

$$
T_{1}=T_{2}=\left(\begin{array}{cc}
I & I \\
I & -I
\end{array}\right), \mathcal{N}_{1}=\mathcal{N}_{2}=\{(\lambda I, \lambda I) \mid \lambda>0\} .
$$

$\mathcal{N}_{1}$ and $\mathcal{N}_{2}$ do not have to be the set of scalings of a matrix pair as in the examples above. For instance, for the nonlinearity whose Jacobian is confined within a polytope or a cone, $\mathcal{N}_{1}$ that satisfies Assumption 1 (or $\mathcal{N}_{2}$ that satisfies Assumption 2) is characterized via matrix inequalities (see Section 5 in [48] for more details). Furthermore, $T_{1}$ does not necessarily has to be chosen to be equal to $T_{2}$.

Because $T_{1}$ in Assumption 1 and $T_{2}$ in Assumption 2 are invertible, the matrix $\Gamma_{i 1}(i=1,2)$ defined as

$$
\Gamma_{i 1}=T_{i 1}-T_{i 2} T_{i 4}^{-1} T_{13}
$$


is also invertible by the matrix inversion lemma. Furthermore, we define the matrix $\Gamma_{i 2}(i=1,2)$ as

$$
\Gamma_{i 2}=T_{i 2} T_{i 4}^{-1} \text {. }
$$

The following theorem provides sufficient conditions for the design of matrices $L_{1}, L_{2}$ in the observer (11) and matrices $K_{1}, K_{2}$ in the controller 12 , when the $\delta$-MM can be parameterized in a block diagonal manner.

Theorem 1. Consider the system described by (1)-(2) with $p(\mathbf{0})=\mathbf{0}$. Suppose that

1) Assumption 1 holds;

2) Assumption 2] holds with $M_{2}=\left(\begin{array}{ll}M_{21} & M_{22} \\ M_{23} & M_{24}\end{array}\right)$ where $M_{24} \in \mathbb{R}^{n_{p} \times n_{p}}$ and $M_{24} \prec 0$;

3) there exist positive numbers $\alpha_{1}, \alpha_{2}, \mu_{1}, \mu_{2}$, matrices $R_{1}, R_{2}, R_{3}, R_{4}$, symmetric and positive definite matrices $P_{1}, P_{2}, X_{1}, X_{2}, Y_{2}$ and a symmetric matrix $Y_{1}$, such that $\left(X_{1}, Y_{1}\right) \in \mathcal{N}_{1},\left(X_{2}, Y_{2}\right) \in \mathcal{N}_{2}$ and

$$
\begin{array}{ccc}
\text { (observer ineq.) } & \left(\begin{array}{cc}
\Phi-\varphi^{\top} Y_{1} \varphi & \phi^{\top} \\
\phi & -X_{1}
\end{array}\right) \preceq 0, \\
\text { (controller ineq.) } & \left(\begin{array}{cc}
\Psi-\varphi^{\top} Y_{2} \varphi & \psi^{\top} \\
\psi & -X_{2}
\end{array}\right) \preceq 0,
\end{array}
$$

where

$$
\begin{aligned}
\Phi & =\left(\begin{array}{ccc}
\Phi_{0} & -P_{1} \tilde{E}_{1} & P_{1} E_{w}+R_{1} \\
* & \mathbf{0} & \mathbf{0} \\
* & * & -\mu_{1} I
\end{array}\right), \\
\Phi_{0} & =\tilde{A}_{1}^{\top} P_{1}+P_{1} \tilde{A}_{1}+C^{\top} R_{1}^{\top}+R_{1} C+\alpha_{1} P_{1}, \\
\Psi & =\left(\begin{array}{ccc}
\Psi_{0} & \tilde{E}_{2} Y_{2}+B R_{4} & E_{w} \\
* & \mathbf{0} & \mathbf{0} \\
* & * & -\mu_{2} I
\end{array}\right), \\
\Psi_{0} & =\tilde{A}_{2} P_{2}+P_{2} \tilde{A}_{2}^{\top}+B R_{3}+R_{3}^{\top} B^{\top}+\alpha_{2} P_{2}, \\
\phi & =\left(-\left(X_{1} \Gamma_{11} C_{q}+R_{2} C\right), X_{1} \Gamma_{12},-R_{2} F_{w}\right), \\
\varphi & =\left(\mathbf{0}_{n_{p} \times n_{x}}, I_{n_{p}}, \mathbf{0}_{n_{p} \times n_{w}}\right), \\
\psi & =\left(\Gamma_{21} C_{q} P_{2}, \Gamma_{22} Y_{2}, \mathbf{0}_{n_{q} \times n_{w}}\right), \\
\tilde{A}_{i} & =A-E T_{i 4}^{-1} T_{i 3} C_{q}, i=1,2, \\
\tilde{E}_{i} & =E T_{i 4}^{-1}, i=1,2,
\end{aligned}
$$

with $\Gamma_{i 1}(i=1,2)$ given in (27) and $\Gamma_{i 2}(i=1,2)$ given in (28). Then, the closed-loop system (15) is ISS w.r.t. $w$ with

$$
\left\{\begin{array}{l}
L_{1}=\Gamma_{11}^{-1} X_{1}^{-1} R_{2} \\
L_{2}=P_{1}^{-1} R_{1}+E T_{14}^{-1} T_{13} L_{1} \\
K_{1}=R_{3} P_{2}^{-1}+K_{2} T_{24}^{-1} T_{23} C_{q} \\
K_{2}=R_{4} Y_{2}^{-1} T_{24} .
\end{array}\right.
$$

Proof. The proof proceeds in five steps.

1) Firstly, we derive dynamics of the system under transformations of variables $q$ and $p$ via $T_{1}$ and $T_{2}$. Since $M_{1}$ (resp. $M_{2}$ ) satisfies Assumption 1 (resp. Assumption 2) with an invertible matrix $T_{1}$ (resp. $T_{2}$ ), we introduce variable transformations from $(q, p)$ to $\left(\tilde{q}_{i}, \tilde{p}_{i}\right)$ as follows:

$$
\left(\begin{array}{c}
\tilde{q}_{i} \\
\tilde{p}_{i}
\end{array}\right)=T_{i}\left(\begin{array}{c}
q \\
p
\end{array}\right) \text {. }
$$

Since $\tilde{p}_{i}=T_{i 3} q+T_{i 4} p$ and $T_{i 4}$ is invertible, we have $p=$ $T_{i 4}^{-1} \tilde{p}_{i}-T_{i 4}^{-1} T_{i 3} q$ and $\tilde{q}_{i}=\Gamma_{i 1} q+\Gamma_{i 2} \tilde{p}_{i}$ for $i=1,2$, where $\Gamma_{i 1}, \Gamma_{i 2}$ are given in 27, 28, Recall that $\Gamma_{i 1}$ is invertible since $T_{i}$ is invertible.

Substituting $p=T_{24}^{-1} \tilde{p}_{2}-T_{24}^{-1} T_{23} q$ into 15 , we have

$$
\dot{x}=\left(\tilde{A}_{2}+B \tilde{K}_{1}\right) x+\left(\tilde{E}_{2}+B \tilde{K}_{2}\right) \tilde{p}_{2}-B \Delta k+E_{w} w,
$$

where $\tilde{p}_{2}=\tilde{p}_{2}\left(C_{q} x\right), \tilde{A}_{2}$ is given in (38), $\tilde{E}_{2}$ is given in (39),

$$
\tilde{K}_{1}=K_{1}-K_{2} T_{24}^{-1} T_{23} C_{q}, \quad \tilde{K}_{2}=K_{2} T_{24}^{-1},
$$

and

$$
\Delta k=\tilde{K}_{1} e-\tilde{K}_{2} \Delta \tilde{p}
$$

where

$$
\Delta \tilde{p}=\tilde{p}_{2}\left(C_{q} \hat{x}\right)-\tilde{p}_{2}\left(C_{q} x\right)
$$

Define $\left(\begin{array}{c}\delta \tilde{q}_{1} \\ \delta \tilde{p}_{1}\end{array}\right)=T_{1}\left(\begin{array}{c}\delta q \\ \delta p\end{array}\right)$. Then, $\delta \tilde{p}_{1}=T_{13} \delta q+T_{14} \delta p$, which implies that $\delta p=T_{14}^{-1} \delta \tilde{p}_{1}-T_{14}^{-1} T_{13} \delta q$. Substituting this form of $\delta p$ and (16) into (15), we have

$$
\dot{e}=\left(\tilde{A}_{1}+\tilde{L}_{2} C\right) e-\tilde{E}_{1} \delta \tilde{p}_{1}+\left(E_{w}+\tilde{L}_{2} F_{w}\right) w,
$$

where $\tilde{A}_{1}$ is given in (38), $\tilde{E}_{1}$ is given in 39 , and $\tilde{L}_{2}$ is defined as

$$
\tilde{L}_{2}=L_{2}-E T_{14}^{-1} T_{13} L_{1}
$$

Equations 42 and (46) are the dynamics of the closed-loop system after transformations of variables via $T_{1}$ and $T_{2}$.

2) We now consider the performance of the observer. From (40) we have $R_{1}=P_{1} \tilde{L}_{2}$ where $\tilde{L}_{2}$ is given in (47), and $R_{2}=X_{1} \Gamma_{11} L_{1}$. Plugging $R_{1}$ into $\Phi$ in (31), we have $\Phi_{0}=P_{1}\left(\tilde{A}_{1}+\tilde{L}_{2} C\right)+\left(\tilde{A}_{1}+\tilde{L}_{2} C\right)^{\top} P_{1}+\alpha_{1} P_{1}$, and the $(1,3)$ entry of $\Phi$ to be $P_{1} E_{w}+R_{1} F_{w}=P_{1}\left(E_{w}+\tilde{L}_{2} F_{w}\right)$; plugging $R_{2}$ into $\phi$ in 35 we have $\phi=X_{1} \phi_{0}$ where $\phi_{0}:=\left(-\Gamma_{11}\left(C_{q}+L_{1} C\right), \Gamma_{12},-\Gamma_{11} L_{1} F_{w}\right)$. Recalling $\varphi$ given in (36) and applying Schur's complement to (29), we have

$$
\Phi+\left(\begin{array}{c}
\phi_{0} \\
\varphi
\end{array}\right)^{\top} \tilde{M}_{1}\left(\begin{array}{c}
\phi_{0} \\
\varphi
\end{array}\right) \preceq 0 .
$$

Define $\xi_{1}=\left(e^{\top}, \delta \tilde{p}_{1}^{\top}, w^{\top}\right)^{\top}$. Pre- and post-multiplying the inequality 48 by $\xi_{1}^{\top}$ and $\xi_{1}$, respectively, we have

$$
\xi_{1}^{\top} \Phi \xi_{1}+\xi_{1}^{\top}\left(\begin{array}{c}
\phi_{0} \\
\varphi
\end{array}\right)^{\top} \tilde{M}_{1}\left(\begin{array}{c}
\phi_{0} \\
\varphi
\end{array}\right) \xi_{1} \leq 0 .
$$

Note that $\delta \tilde{q}_{1}=T_{11} \delta q+T_{12} \delta p=T_{11} \delta q+T_{12} T_{14}^{-1} \delta \tilde{p}_{1}$ $T_{12} T_{14}^{-1} T_{13} \delta q=\Gamma_{11} \delta q+\Gamma_{12} \delta \tilde{p}_{1}=-\Gamma_{11}\left(C_{q}+L_{1} C\right) e+$ $\Gamma_{12} \delta \tilde{p}_{1}-\Gamma_{11} L_{1} F_{w} w$. Therefore, $\left(\begin{array}{c}\delta \tilde{q}_{1} \\ \delta \tilde{p}_{1}\end{array}\right)=\left(\begin{array}{c}\phi_{0} \\ \varphi\end{array}\right) \xi_{1}$. Since $\left(\begin{array}{l}\delta q \\ \delta p\end{array}\right)^{\top} M\left(\begin{array}{l}\delta q \\ \delta p\end{array}\right) \geq 0$, we have $\left(\begin{array}{l}\delta \tilde{q}_{1} \\ \delta \tilde{p}_{1}\end{array}\right)^{\top} \tilde{M}_{1}\left(\begin{array}{l}\delta \tilde{q}_{1} \\ \delta \tilde{p}_{1}\end{array}\right) \geq 0$, and therefore, $\xi_{1}^{\top}\left(\begin{array}{c}\phi_{0} \\ \varphi\end{array}\right)^{\top} \tilde{M}_{1}\left(\begin{array}{c}\phi_{0} \\ \varphi\end{array}\right) \xi_{1} \geq 0$. Thus, $\xi_{1}^{\top} \Phi \xi_{1} \leq 0$ from (49), which is equivalent to $2 e^{\top} P_{1}\left[\left(\tilde{A}+\tilde{L}_{2} \tilde{C}\right) e-\tilde{E}_{1} \delta \tilde{p}_{1}+\right.$ $\left.\left(E_{w}+L_{2} F_{w}\right) w\right]+\alpha_{1} e^{\top} P_{1} e-\mu_{1}\|w\|^{2} \leq 0$.

Define $V_{1}(e)=e^{\top} P_{1} e$. Then the derivative of $V_{1}$ along the 
trajectory of 46 satisfies

$$
\begin{aligned}
\dot{V}_{1} & =2 e^{\top} P_{1}\left[\left(\tilde{A}+\tilde{L}_{2} \tilde{C}\right) e-\tilde{E}_{1} \delta \tilde{p}_{1}+\left(E_{w}+\tilde{L}_{2} F_{w}\right) w\right] \\
& \leq-\alpha_{1} e^{\top} P_{1} e+\mu_{1}\|w\|^{2} .
\end{aligned}
$$

3) We now prove that $\|\Delta k\| /\|e\|$ is bounded where $\Delta k$ is given in (44). Since $M_{24}=T_{22}^{\top} X_{2}^{-1} T_{22}-T_{24}^{\top} Y_{2}^{-1} T_{24} \prec 0$ and $T_{24}$ is invertible, we have

$$
\Gamma_{22}^{\top} X_{2}^{-1} \Gamma_{22}-Y_{2}^{-1}=T_{24}^{-\top} M_{24} T_{24}^{-1} \prec 0 .
$$

Recall that $\Delta q=-C_{q} e$ in 21) and define $\Delta \tilde{q}:=\tilde{q}_{2}\left(C_{q} \hat{x}\right)-$ $\tilde{q}_{2}\left(C_{q} x\right)$. Then, $\Delta \tilde{q}=-\Gamma_{21} C_{q} e+\Gamma_{22} \Delta \tilde{p}$ where $\Delta \tilde{p}$ is given in (45). Define $\zeta=\left(e^{\top}, \Delta \tilde{p}^{\top}\right)^{\top}$. Therefore,

$$
\begin{aligned}
& \zeta^{\top}\left(\begin{array}{cc}
-\Gamma_{21} C_{q} & \Gamma_{22} \\
\mathbf{0} & I
\end{array}\right)^{\top} \tilde{M}_{2}\left(\begin{array}{cc}
-\Gamma_{21} C_{q} & \Gamma_{22} \\
\mathbf{0} & I
\end{array}\right) \zeta \\
= & \left(\begin{array}{c}
-\Gamma_{21} C_{q} e+\Gamma_{22} \Delta \tilde{p} \\
\Delta \tilde{p}
\end{array}\right)^{\top} \tilde{M}_{2}\left(\begin{array}{c}
-\Gamma_{21} C_{q} e+\Gamma_{22} \Delta \tilde{p} \\
\Delta \tilde{p}
\end{array}\right) \\
= & \left(\begin{array}{c}
\Delta q \\
\Delta p
\end{array}\right)^{\top} T_{2}^{\top} \tilde{M}_{2} T_{2}\left(\begin{array}{c}
\Delta q \\
\Delta p
\end{array}\right) \geq 0,
\end{aligned}
$$

where the last equality is from 26 in Assumption 2 Hence, $e^{\top} C_{q}^{\top} \Gamma_{21}^{\top} X_{2}^{-1} \Gamma_{21} C_{q} e-2 e^{\top} C_{q}^{\top} \Gamma_{21}^{\top} X_{2}^{-1} \Gamma_{22} \Delta \tilde{p}+$ $\Delta \tilde{p}^{\top}\left(\Gamma_{22}^{\top} X_{2}^{-1} \Gamma_{22}-Y_{2}^{-1}\right) \Delta \tilde{p} \geq 0$. From (51), the inequality above implies that $\kappa_{1}\|e\|^{2}+\kappa_{2}\|e\|\|\Delta \tilde{p}\|-\kappa_{3}\|\Delta \tilde{p}\|^{2} \geq 0$ where $\kappa_{1}=\lambda_{\max }\left(C_{q}^{\top} \Gamma_{21}^{\top} X_{2}^{-1} \Gamma_{21} C_{q}\right), \kappa_{2}=2\left\|C_{q}^{\top} \Gamma_{21}^{\top} X_{2}^{-1} \Gamma_{22}\right\|$, $\kappa_{3}=\lambda_{\min }\left(Y_{2}^{-1}-\Gamma_{22}^{\top} X_{2}^{-1} \Gamma_{22}\right)$. Clearly, $\kappa_{1}, \kappa_{3}>0, \kappa_{2} \geq 0$. Therefore, we have $\|\Delta \tilde{p}\| \leq \kappa\|e\|$ where $\kappa:=\left(\kappa_{2}+\right.$ $\left.\sqrt{\kappa_{2}^{2}+4 \kappa_{1} \kappa_{3}}\right) / 2 \kappa_{3}>0$.

Since $\Delta k=\tilde{K}_{1} e+\tilde{K}_{2} \Delta \tilde{p}$ by [44], we have

$$
\|\Delta k\| \leq \hat{\kappa}\|e\|
$$

for all $x, e$, where $\hat{\kappa}=\left\|\tilde{K}_{1}\right\|+\left\|\tilde{K}_{2}\right\| \kappa>0$, which bounds $\|\Delta k\| /\|e\|$.

4) Next, we analyse controller performance. From (40) we have $R_{3}=\tilde{K}_{1} P_{2}$ and $R_{4}=\tilde{K}_{2} Y_{2}$ where $\tilde{K}_{1}, \tilde{K}_{2}$ are given in (43). Plugging $R_{3}, R_{4}$ into (33), we have $\Psi_{0}=\left(\tilde{A}_{2}+\right.$ $\left.B \tilde{K}_{1}\right) P_{2}+P_{2}\left(\tilde{A}_{2}+B \tilde{K}_{1}\right)^{\top}+\alpha_{2} P_{2}$, and the $(1,2)$ entry of $\Psi$ to be $\left(\tilde{E}_{2}+B \tilde{K}_{2}\right) Y_{2}$. Pre- and post-multiplying the inequality (30) by the matrix $\operatorname{diag}\left(I_{n}, Y_{2}^{-1}, I_{n_{w}}, I_{n_{q}}\right)$, and then applying Schur's complement, we have

$$
\tilde{\Psi}+\left(\begin{array}{c}
\psi_{1} \\
\varphi
\end{array}\right)^{\top} \tilde{M}_{2}\left(\begin{array}{c}
\psi_{1} \\
\varphi
\end{array}\right) \preceq 0
$$

where

$$
\tilde{\Psi}=\left(\begin{array}{ccc}
\Psi_{0} & \tilde{E}_{2}+B \tilde{K}_{2} & E_{w} \\
* & \mathbf{0} & \mathbf{0} \\
* & * & -\mu_{2} I
\end{array}\right),
$$

and $\psi_{1}=\left(\Gamma_{21} C_{q} P_{2}, \Gamma_{22}, \mathbf{0}_{n_{q} \times n_{w}}\right), \Psi_{0}$ is shown above, $\varphi$ is given in (36). Let $P_{3}=P_{2}^{-1}$ and pre- and post-multiply the inequality (53) by $\operatorname{diag}\left(P_{3}, I_{n_{p}}, I_{n_{w}}\right)$ and its transpose, respectively. This results in

$$
\hat{\Psi}+\left(\begin{array}{c}
\psi_{0} \\
\varphi
\end{array}\right)^{\top} \tilde{M}_{2}\left(\begin{array}{c}
\psi_{0} \\
\varphi
\end{array}\right) \preceq 0
$$

where $\psi_{0}=\left(\Gamma_{21} C_{q}, \Gamma_{22}, \mathbf{0}_{n_{q} \times n_{w}}\right)$ and

$$
\begin{aligned}
\hat{\Psi} & =\left(\begin{array}{ccc}
\hat{\Psi}_{0} & P_{3}\left(\tilde{E}_{2}+B \tilde{K}_{2}\right) & P_{3} E_{w} \\
* & \mathbf{0} & \mathbf{0} \\
* & * & -\mu_{2} I
\end{array}\right), \\
\hat{\Psi}_{0} & =P_{3}\left(\tilde{A}_{2}+B \tilde{K}_{1}\right)+\left(\tilde{A}_{2}+B \tilde{K}_{1}\right)^{\top} P_{3}+\alpha_{2} P_{3} .
\end{aligned}
$$

Define $\xi_{2}=\left(x^{\top}, \tilde{p}_{2}^{\top}, w^{\top}\right)^{\top}$. Pre- and post-multiplying the inequality (54) by $\xi_{2}^{\top}$ and $\xi_{2}$, respectively, we have

$$
\xi_{2}^{\top} \hat{\Psi} \xi_{2}+\xi_{2}^{\top}\left(\begin{array}{c}
\psi_{0} \\
\varphi
\end{array}\right)^{\top} \tilde{M}_{2}\left(\begin{array}{c}
\psi_{0} \\
\varphi
\end{array}\right) \xi_{2} \leq 0 .
$$

By 26, and 41, we have $\left(\begin{array}{c}\tilde{q}_{2} \\ \tilde{p}_{2}\end{array}\right)^{\top} \tilde{M}_{2}\left(\begin{array}{c}\tilde{q}_{2} \\ \tilde{p}_{2}\end{array}\right) \geq 0$. Since $\tilde{q}_{2}=\Gamma_{21} q+\Gamma_{22} \tilde{p}_{2}=\Gamma_{21} C_{q} x+\Gamma_{22} \tilde{p}_{2},\left(\begin{array}{c}\tilde{q}_{2} \\ \tilde{p}_{2}\end{array}\right)=\left(\begin{array}{c}\psi_{0} \\ \varphi\end{array}\right) \xi_{2}$, $\xi_{2}^{\top}\left(\begin{array}{c}\psi_{0} \\ \varphi\end{array}\right)^{\top} \tilde{M}_{2}\left(\begin{array}{c}\psi_{0} \\ \varphi\end{array}\right) \xi_{2} \geq 0$. Thus, $\xi_{2}^{\top} \hat{\Psi} \xi_{2} \leq 0$ from [57], which is equivalent to $2 x^{\top} P_{3}\left[\left(\tilde{A}_{2}+B \tilde{K}_{1}\right) x+\left(\tilde{E}_{2}+B \tilde{K}_{2}\right) \tilde{p}_{2}+\right.$ $\left.E_{w} w\right]+\alpha_{2} x^{\top} P_{3} x-\mu_{2}\|w\|^{2} \leq 0$.

Let $V_{2}(x)=x^{\top} P_{3} x$. Then the derivative of $V_{2}$ along the trajectory of (42) satisfies

$$
\begin{aligned}
\dot{V}_{2} & =2 x^{\top} P_{3}\left[\left(\tilde{A}_{2}+B \tilde{K}_{1}\right) x+\left(\tilde{E}_{2}+B \tilde{K}_{2}\right) \tilde{p}_{2}-B \Delta k+E_{w} w\right] \\
& \leq-\alpha_{2} x^{\top} P_{3} x+\mu_{2}\|w\|^{2}+2\left\|P_{3} B\right\|\|x\|\|\Delta k\| .
\end{aligned}
$$

Recalling [52], we have

$$
\dot{V}_{2} \leq-\alpha_{2} x^{\top} P_{3} x+\mu_{2}\|w\|^{2}+\theta\|x\|\|e\| .
$$

where $\theta=2\left\|P_{3} B\right\| \hat{\kappa}$.

5) Finally, we prove that the closed-loop system expressed by (42) and 46 is ISS with respect to $w$. Choose two constants $c_{1}, c_{2}$ as $c_{1}=\alpha_{1} \lambda_{m}\left(P_{1}\right) / \lambda_{M}\left(P_{1}\right), \quad c_{2}=$ $\alpha_{1} \lambda_{m}\left(P_{3}\right) / \lambda_{M}\left(P_{3}\right)$. Since $c_{1}>0, c_{2}>0$, we can choose two constants $\alpha_{0}>0, \beta_{0}>0$ such that $\alpha_{0}<\min \left\{c_{1}, c_{2}\right\}, \beta_{0} \geq$ $\frac{\theta^{2}}{4 \lambda_{M}\left(P_{1}\right) \lambda_{M}\left(P_{3}\right)\left(c_{1}-\alpha_{0}\right)\left(c_{2}-\alpha_{0}\right)}$. Then, it is easy to check that the matrix $P_{0}:=\left(\begin{array}{cc}\tilde{P}_{0} & \theta / 2 \\ \theta / 2 & \hat{P}_{0}\end{array}\right)$ is negative semi-definite where $\tilde{P}_{0}=-\alpha_{2} \lambda_{m}\left(P_{3}\right)+\alpha_{0} \lambda_{M}\left(P_{3}\right)$ and $\hat{P}_{0}=\beta_{0}\left(-\alpha_{1} \lambda_{m}\left(P_{1}\right)+\right.$ $\left.\alpha_{0} \lambda_{M}\left(P_{1}\right)\right)$. Define a matrix $P$ as $P=\left(\begin{array}{cc}P_{3} & \mathbf{0} \\ \mathbf{0} & \beta_{0} P_{1}\end{array}\right)$. Clearly, $P$ is positive definite. We can verify that the candidate Lyapunov function $V(x, e):=z^{\top} P z$ satisfies $V(x, e)=$ $\beta_{0} V_{1}(e)+V_{2}(x)$, and its derivative along the trajectory of (42) and (46) satisfies

$$
\begin{aligned}
\dot{V}+\alpha_{0} V & \leq-\alpha_{1} \beta_{0} e^{\top} P_{1} e-\alpha_{2} x^{\top} P_{3} x+\theta\|x\|\|e\|+\alpha_{0} V \\
& \leq(\|x\|,\|e\|) P_{0}(\|x\|,\|e\|)^{\top}+\left(\mu_{1} \beta_{0}+\mu_{2}\right)\|w\|^{2} \\
& \leq\left(\mu_{1} \beta_{0}+\mu_{2}\right)\|w\|^{2} .
\end{aligned}
$$

Therefore, the closed-loop system (42) and 46, or equivalently (15), satisfies (8) with $\mathcal{K}_{\infty}$ functions $\gamma(\|(x, e)\|)=$ $-\alpha_{0} \lambda_{m}(P)\|(x, e)\|^{2}$ and $\chi(\|w\|)=\left(\mu_{1} \beta_{0}+\mu_{2}\right)\|w\|^{2}$. This completes the proof.

Remark 3. In the proof of Theorem 1] we first prove that the observer error $e$ is ISS w.r.t. $w$ (see (50) in step 2), then the state $x$ is ISS w.r.t. $w$ and e (see (58) in step 4), 
and finally $(x, e)$ is ISS w.r.t. $w$ (see (59) in step 5). This procedure of proving global stabilization is similar to the certainty equivalence proof used in [25].

Remark 4. If $\alpha_{1}$ is fixed, then (29) is an LMI in decision variables $\mu_{1}, P_{1}, R_{1}, R_{2}, X_{1}, Y_{1}$ that are used to determine observer gains $L_{1}, L_{2}$; if $\alpha_{2}$ is fixed, then (30) is an LMI in decision variables $\mu_{2}, P_{2}, R_{3}, R_{4}, X_{2}, Y_{2}$ that are used to determine controller gains $K_{1}, K_{2}$. There is no coupling in decision variables in LMIs (29) and (30), implying a separation of the controller and observer designs.

Remark 5. The proof of Theorem 1 indicates that larger $\alpha_{1}, \alpha_{2}$ result in a larger function $\gamma(\cdot) \in \mathcal{K}_{\infty}$ in (8), which in turn indicates a faster convergence rate for the system (17). The convergence rate guarantee given in the proof of Theorem 1. $\alpha_{0}$, can be improved by finding a new ISS-Lyapunov function $V=z^{\top} P z$ via Proposition 2

Remark 6. The condition $M_{24} \prec 0$ in Theorem 1 is used to prove that $\|\Delta k\| /\|e\|$ is bounded, which holds automatically when $p$ is globally Lipschitz. The condition $M_{24} \prec 0$ can be replaced by a growth condition on $p$ similar to Theorem 2 of [28]. Specifically, for a system described by (1)-(2) where $E_{w}=F_{w}=0$, if all the conditions of Theorem 1 but $M_{24} \prec 0$ hold, and there exist a $\mathcal{K}$ function $g_{1}$ and a non-decreasing function $g_{2}:[0, \infty) \rightarrow[0, \infty)$ such that $\left\|p\left(C_{q}(x+\Delta x)\right)-p\left(C_{q} x\right)\right\| \leq g_{1}(\|\Delta x\|)\left\|C_{q} x\right\|$ for all $x, \Delta x$ that satisfy $\left\|C_{q} x\right\| \geq g_{2}(\|\Delta x\|)$, then the feedback controller (12) with $L_{1}, L_{2}, K_{1}, K_{2}$ given by (40) renders the closed-loop system (15) globally exponentially stable.

The condition $M_{24} \prec 0$ can be eliminated by using a simpler form of $u$. Specifically, suppose that the observerbased controller $u$ has the form $u(t)=K_{1} \hat{x}(t)$, all the conditions of Theorem 1 but $M_{24} \prec 0$ hold with $R_{4}=\mathbf{0}$, and $L_{1}, L_{2}, K_{1}$ are given by (40), then the closed-loop system (15) is ISS w.r.t. w. In this case, the LMI (30) is less likely to be satisfied by fixing $R_{4}=\mathbf{0}$.

\section{B. Block Anti-Triangular Parameterization}

In this subsection, we consider a block anti-triangular parameterization of the $\delta$-MM for $p$. The following assumption on the parameterization of $M$ is given first.

Assumption 3. There exist a set $\mathcal{N}$ of matrix pairs $(X, Y)$ with $X \in \mathbb{R}^{n_{q} \times n_{p}}, Y \in \mathbb{R}^{n_{p} \times n_{p}}$, and an invertible matrix $T \in \mathbb{R}^{\left(n_{p}+n_{q}\right) \times\left(n_{p}+n_{q}\right)}$, such that $M$ given below is a $\delta$-MM of $p$ for all $(X, Y) \in \mathcal{N}$ :

$$
M=T^{\top} \tilde{M} T \text { where } \tilde{M}=\left(\begin{array}{cc}
\mathbf{0} & X \\
X^{\top} & Y
\end{array}\right) .
$$

The following theorem provides sufficient conditions for the design of matrices $L_{1}, L_{2}, K_{1}, K_{2}$ when the $\delta$-MM $M$ can be parameterized in a block anti-triangular manner.

Theorem 2. Consider the system described by (1)-(2) with $p(\mathbf{0})=\mathbf{0}$. Suppose that

1) Assumption 3 holds for some $T_{1}, \mathcal{N}_{1}$ and $T_{2}, \mathcal{N}_{2}$, respectively, where $T_{1}$ and $T_{2}$ are partitioned as in (23) and in (25), respectively, with $T_{14}, T_{24}$ invertible;
2) there exist positive constants $\alpha_{1}, \alpha_{2}, \mu_{1}, \mu_{2}$, matrices $R_{1}, R_{2}, R_{3}, R_{4}, X_{1}, Y_{1} . X_{2}, Y_{2}$, and symmetric and positive definite matrices $P_{1}, P_{2}$, such that $\left(X_{1}, Y_{1}\right) \in \mathcal{N}_{1},\left(X_{2}, Y_{2}\right) \in$ $\mathcal{N}_{2}$, and

$$
\begin{aligned}
& \Phi+\Upsilon_{1}^{\top} \tilde{M}_{1} \Upsilon_{1}+\Upsilon_{2}^{\top} \Upsilon_{1}+\Upsilon_{1}^{\top} \Upsilon_{2} \preceq 0, \\
& \Psi+\Upsilon_{3}^{\top} \tilde{M}_{2} \Upsilon_{3}+\Upsilon_{4}^{\top} \Upsilon_{3}+\Upsilon_{3}^{\top} \Upsilon_{4} \preceq 0, \\
& \Gamma_{12}^{\top} X_{1}+X_{1}^{\top} \Gamma_{12}+Y_{1} \prec 0,
\end{aligned}
$$

where

$$
\begin{aligned}
& \tilde{M}_{1}=\left(\begin{array}{cc}
\mathbf{0} & X_{1} \\
X_{1}^{\top} & Y_{1}
\end{array}\right), \tilde{M}_{2}=\left(\begin{array}{cc}
\mathbf{0} & X_{2} \\
X_{2}^{\top} & Y_{2}
\end{array}\right), \\
& \Psi=\left(\begin{array}{ccc}
\Psi_{0} & \tilde{E}_{2}+B R_{4} & E_{w} \\
* & \mathbf{0} & \mathbf{0} \\
* & * & -\mu_{2} I
\end{array}\right), \\
& \left\{\begin{array}{l}
\Upsilon_{1}=\left(\begin{array}{ccc}
-\Gamma_{11} C_{q} & \Gamma_{12} & \mathbf{0}_{n_{q} \times n_{w}} \\
\mathbf{0}_{n_{p} \times n_{x}} & I_{n_{p}} & \mathbf{0}_{n_{p} \times n_{w}}
\end{array}\right), \\
\Upsilon_{2}=\left(\begin{array}{ccc}
\mathbf{0}_{n_{q} \times n_{x}} & \mathbf{0}_{n_{q} \times n_{p}} & \mathbf{0}_{n_{q} \times n_{w}} \\
-R_{2} C & \mathbf{0}_{n_{p}} & -R_{2} F_{w}
\end{array}\right), \\
\Upsilon_{3}=\left(\begin{array}{ccc}
\mathbf{0}_{n_{q} \times n_{x}} & \Gamma_{22} & \mathbf{0}_{n_{q} \times n_{w}} \\
\mathbf{0}_{n_{p} \times n_{x}} & I_{n_{p}} & \mathbf{0}_{n_{p} \times n_{w}}
\end{array}\right), \\
\Upsilon_{4}=\left(\begin{array}{ccc}
\mathbf{0}_{n_{q} \times n_{x}} & \mathbf{0}_{n_{q} \times n_{p}} & \mathbf{0}_{n_{q} \times n_{w}} \\
X_{2} \Gamma_{21} C_{q} P_{2} & \mathbf{0}_{n_{p}} & \mathbf{0}_{n_{p} \times n_{w}}
\end{array}\right),
\end{array}\right.
\end{aligned}
$$

with $\Gamma_{i 1}(i=1,2)$ given in 277, $\Gamma_{i 2}(i=1,2)$ given in (28), $\Phi$ given in (31), $\Phi_{0}$ given in (32), $\Psi_{0}$ given in (34), $\varphi$ given in (36), $\tilde{A}_{i}(i=1,2)$ given in (38) and $\tilde{E}_{i}(i=1,2)$ given in (39). If $X_{1}$ has full row rank, then the closed-loop system (15) is ISS w.r.t. $w$ with $L_{2}, K_{1}$ given by (40), and $L_{1}, K_{2}$ given by

$$
L_{1}=\Gamma_{11}^{-1} X_{1}^{\dagger} R_{2}, \quad K_{2}=R_{4} T_{24},
$$

where $X_{1}^{\dagger}$ is the right inverse of $X_{1}$.

Proof. As shown in (42) and 46, dynamics of the closed-loop system under transformations can be described as

$$
\begin{aligned}
\dot{x} & =\left(\tilde{A}_{2}+B \tilde{K}_{1}\right) x+\left(\tilde{E}_{2}+B \tilde{K}_{2}\right) \tilde{p}_{2}-B \Delta k+E_{w} w, \\
\dot{e} & =\left(\tilde{A}_{1}+\tilde{L}_{2} C\right) e-\tilde{E}_{1} \delta \tilde{p}_{1}+\left(E_{w}+\tilde{L}_{2} F_{w}\right) w,
\end{aligned}
$$

where $\tilde{K}_{1}, \tilde{K}_{2}$ are given in (43), $\Delta k$ is given in (44), $\tilde{p}_{2}$ is given in (41), and $\tilde{L}_{2}$ is given in (47). From (40) and (67), we have $R_{1}=P_{1} \tilde{L}_{2}$ and $R_{2}=X_{1} \Gamma_{11} L_{1}$. We claim that (61) is equivalent to

$$
\Phi+Q_{1}^{\top} \tilde{M}_{1} Q_{1} \preceq 0
$$

and 62 is equivalent to

$$
\Psi+Q_{2}^{\top} \tilde{M}_{2} Q_{2} \preceq 0
$$

where

$$
\begin{aligned}
Q_{1} & =\left(\begin{array}{ccc}
-\Gamma_{11}\left(C_{q}+L_{1} C\right) & \Gamma_{12} & -\Gamma_{11} L_{1} F_{w} \\
\mathbf{0}_{n_{p} \times n_{x}} & I_{n_{p}} & \mathbf{0}_{n_{p} \times n_{w}}
\end{array}\right), \\
Q_{2} & =\left(\begin{array}{ccc}
\Gamma_{21} C_{q} P_{2} & \Gamma_{22} & \mathbf{0}_{n_{q} \times n_{w}} \\
\mathbf{0}_{n_{p} \times n_{x}} & I_{n_{p}} & \mathbf{0}_{n_{p} \times n_{w}}
\end{array}\right) .
\end{aligned}
$$


Indeed, $Q_{1}$ can be written as $Q_{1}=\Upsilon_{1}+\hat{\Upsilon}_{2}$ where $\Upsilon_{1}$ is given in 66 and

$$
\hat{\Upsilon}_{2}=\left(\begin{array}{ccc}
-\Gamma_{11} L_{1} C & \mathbf{0}_{n_{q} \times n_{p}} & -\Gamma_{11} L_{1} F_{w} \\
\mathbf{0}_{n_{p} \times n_{x}} & \mathbf{0}_{n_{p} \times n_{p}} & \mathbf{0}_{n_{p} \times n_{w}}
\end{array}\right) .
$$

It is easy to verify that $\Upsilon_{2}=\tilde{M}_{1} \hat{\Upsilon}_{2}$ and $\hat{\Upsilon}_{2}^{\top} \tilde{M}_{1} \hat{\Upsilon}_{2}=\mathbf{0}$. Therefore,

$$
\begin{aligned}
Q_{1}^{\top} \tilde{M}_{1} Q_{1} & =\left(\Upsilon_{1}+\hat{\Upsilon}_{2}\right)^{\top} \tilde{M}_{1}\left(\Upsilon_{1}+\hat{\Upsilon}_{2}\right) \\
& =\Upsilon_{1}^{\top} \tilde{M}_{1} \Upsilon_{1}+\hat{\Upsilon}_{2}^{\top} \tilde{M}_{1} \Upsilon_{1}+\Upsilon_{1}^{\top} \tilde{M}_{1} \hat{\Upsilon}_{2}+\hat{\Upsilon}_{2}^{\top} \tilde{M}_{1} \hat{\Upsilon}_{2} \\
& =\Upsilon_{1}^{\top} \tilde{M}_{1} \Upsilon_{1}+\Upsilon_{2}^{\top} \Upsilon_{1}+\Upsilon_{1}^{\top} \Upsilon_{2} .
\end{aligned}
$$

Similarly, $Q_{2}$ can be written as $Q_{2}=\Upsilon_{3}+\hat{\Upsilon}_{4}$ where $\Upsilon_{3}$ is given in 66 and

$$
\hat{\Upsilon}_{4}=\left(\begin{array}{ccc}
\Gamma_{21} C_{q} P_{2} & \mathbf{0}_{n_{q} \times n_{p}} & \mathbf{0}_{n_{q} \times n_{w}} \\
\mathbf{0}_{n_{p} \times n_{x}} & \mathbf{0}_{n_{p} \times n_{p}} & \mathbf{0}_{n_{p} \times n_{w}}
\end{array}\right) .
$$

It is easy to verify that $\Upsilon_{4}=\tilde{M}_{2} \hat{\Upsilon}_{4}$ and $\hat{\Upsilon}_{4}^{\top} \tilde{M}_{2} \hat{\Upsilon}_{4}=$ 0. Therefore, $Q_{2}^{\top} \tilde{M}_{2} Q_{2}=\left(\Upsilon_{3}+\hat{\Upsilon}_{4}\right)^{\top} \tilde{M}_{2}\left(\Upsilon_{3}+\hat{\Upsilon}_{4}\right)=$ $\Upsilon_{3}^{\top} \tilde{M}_{2} \Upsilon_{3}+\Upsilon_{3}^{\top} \Upsilon_{4}+\Upsilon_{4}^{\top} \Upsilon_{3}$. Hence, our claim is proved.

Plugging $R_{1}$ into $\Phi_{0}$ and $\Phi$, we have $\Phi_{0}=P_{1}\left(\tilde{A}_{1}+\right.$ $\left.\tilde{L}_{2} C\right)+\left(\tilde{A}_{1}+\tilde{L}_{2} C\right)^{\top} P_{1}+\alpha_{1} P_{1}$, and the $(1,3)$ entry of $\Phi$ is $P_{1}\left(E_{w}+\tilde{L}_{2} F_{w}\right)$. Define $\xi_{1}=\left(e^{\top}, \delta \tilde{p}_{1}^{\top}, w^{\top}\right)^{\top}$. Pre- and postmultiplying (68) by $\xi_{1}^{\top}$ and $\xi_{1}$, respectively, we have $\xi_{1}^{\top} \Phi \xi_{1}+$ $\xi_{1}^{\top} Q_{1}^{\top} \tilde{M}_{1} Q_{1} \xi_{1} \leq 0$. Since $Q_{1} \xi_{1}=\left(\begin{array}{l}\delta \tilde{q}_{1} \\ \delta \tilde{p}_{1}\end{array}\right)=T_{1}\left(\begin{array}{l}\delta q \\ \delta p\end{array}\right)$ and $M_{1}$ satisfies Assumption 3, we have $\xi_{1}^{\top} Q_{1}^{\top} \tilde{M}_{1} Q_{1} \xi_{1} \geq 0$, which implies that $\xi_{1}^{\top} \Phi \xi_{1} \leq 0$. Hence, $2 e^{\top} P_{1}\left[\left(\tilde{A}+\tilde{L}_{2} \tilde{C}\right) e-\right.$ $\left.\tilde{E}_{1} \delta \tilde{p}_{1}+\left(E_{w}+\tilde{L}_{2} F_{w}\right) w\right]+\alpha_{1} e^{\top} P_{1} e-\mu_{1}\|w\|^{2} \leq 0$. Define $V_{1}(e)=e^{\top} P_{1} e$. Then, we have

$$
\dot{V}_{1} \leq-\alpha_{1} e^{\top} P_{1} e+\mu_{1}\|w\|^{2} .
$$

Define $\Delta q=C_{q} \hat{x}-C_{q} x$ and $\Delta \tilde{q}:=\tilde{q}_{1}\left(C_{q} \hat{x}\right)-\tilde{q}_{1}\left(C_{q} x\right)$. Then, $\Delta q=-C_{q} e$ and $\Delta \tilde{q}=-\Gamma_{11} C_{q} e+\Gamma_{12} \Delta \tilde{p}$ where $\Delta \tilde{p}=$ $\tilde{p}_{1}\left(C_{q} \hat{x}\right)-\tilde{p}_{1}\left(C_{q} x\right)$. Define $\zeta=\left(e^{\top}, \Delta \tilde{p}^{\top}\right)^{\top}$. Therefore,

$$
\begin{aligned}
& \zeta^{\top}\left(\begin{array}{cc}
-\Gamma_{11} C_{q} & \Gamma_{12} \\
\mathbf{0} & I
\end{array}\right)^{\top} \tilde{M}_{1}\left(\begin{array}{cc}
-\Gamma_{11} C_{q} & \Gamma_{12} \\
\mathbf{0} & I
\end{array}\right) \zeta \\
= & \left(\begin{array}{c}
\Delta q \\
\Delta p
\end{array}\right)^{\top} T_{1}^{\top} \tilde{M}_{1} T_{1}\left(\begin{array}{c}
\Delta q \\
\Delta p
\end{array}\right) \geq 0,
\end{aligned}
$$

where the last equality is from Assumption 3 Hence, $-2 e^{\top} C_{q}^{\top} \Gamma_{11}^{\top} X_{1} \Delta \tilde{p}+\Delta \tilde{p}^{\top}\left(\Gamma_{12}^{\top} X_{1}+X_{1}^{\top} \Gamma_{12}+Y_{1}\right) \Delta \tilde{p} \geq 0$. From (63), the inequality above implies that $\kappa_{1}\|e\|\|\Delta \tilde{p}\|-$ $\kappa_{2}\|\Delta \tilde{p}\|^{2} \geq 0$, where $\kappa_{1}=2\left\|C_{q}^{\top} \Gamma_{11}^{\top} X_{1}\right\|$ and $\kappa_{2}=$ $-\lambda_{\min }\left(\Gamma_{12}^{\top} X_{1}+X_{1}^{\top} \Gamma_{12}+Y_{1}\right)$. Noticing that $\kappa_{1} \geq 0, \kappa_{2}>0$, we have $\|\Delta \tilde{p}\| \leq \frac{\kappa_{1}}{\kappa_{2}}\|e\|$. Noting that $\Delta k=\hat{K}_{1} e+\hat{K}_{2} \Delta \tilde{p}$ with $\Delta \tilde{p}=\tilde{p}_{1}\left(C_{q} \hat{x}\right)-\tilde{p}_{1}\left(C_{q} x\right), \hat{K}_{1}=K_{1}-K_{2} T_{14}^{-1} T_{13} C_{q}$, $\hat{K}_{2}=K_{2} T_{14}^{-1}$, we have $\|\Delta k\| \leq \hat{\kappa}\|e\|$ for all $x, e$, where $\hat{\kappa}=\left\|\tilde{K}_{1}\right\|+\left\|\tilde{K}_{2}\right\| \kappa_{1} / \kappa_{2} \geq 0$.

From (40) and (67) we have $R_{3}=\tilde{K}_{1} P_{2}$ and $R_{4}=\tilde{K}_{2}$ where $\tilde{K}_{1}, K_{2}$ are defined in (43). Plugging $R_{3}, R_{4}$ into $\Psi_{0}$ and $\Psi$, we have $\Psi_{0}=\left(\tilde{A}_{2}+B K_{1}\right) P_{2}+P_{2}\left(\tilde{A}_{2}+B \tilde{K}_{1}\right)^{\top}+$ $\alpha_{2} P_{2}$, and the $(1,2)$ entry of $\Psi$ is $\tilde{E}_{2}+B \tilde{K}_{2}$. Let $P_{3}=P_{2}^{-1}$ and pre- and post-multiply 69 by $\operatorname{diag}\left(P_{3}, I_{n_{p}}, I_{n_{w}}\right)$ and its transpose, respectively. This results in

$$
\hat{\Psi}+Q_{3}^{\top} \tilde{M}_{2} Q_{3} \preceq 0,
$$

where $Q_{3}=\left(\begin{array}{ccc}\Gamma_{21} C_{q} & \Gamma_{22} & \mathbf{0}_{n_{q} \times n_{w}} \\ \mathbf{0}_{n_{p} \times n_{x}} & I_{n_{p}} & \mathbf{0}_{n_{p} \times n_{w}}\end{array}\right)$ and $\hat{\Psi}$ is given in (55) with $\hat{\Psi}_{0}$ given in (56). Define $\xi_{2}=\left(x^{\top}, \tilde{p}_{2}^{\top}, w^{\top}\right)^{\top}$. Pre- and post-multiplying (70) by $\xi_{2}^{\top}$ and $\xi_{2}$, respectively, we have $\xi_{2}^{\top} \hat{\Psi} \xi_{2}+\xi_{2}^{\top} Q_{3}^{\top} \vec{M}_{2} Q_{3} \xi_{2} \leq 0$. Since $Q_{3} \xi_{2}=$ $\left(\begin{array}{c}\tilde{q}_{2} \\ \tilde{p}_{2}\end{array}\right)=T_{2}\left(\begin{array}{l}q \\ p\end{array}\right)$ and $M_{2}$ satisfies Assumption 3 it follows that $\xi_{2}^{\top} Q_{3}^{\top} \tilde{M}_{2} Q_{3} \xi_{2} \geq 0$. Hence, we have $\xi_{2}^{\top} \hat{\Psi} \xi_{2} \leq 0$, which is equivalent to $2 x^{\top} P_{3}\left[\left(\tilde{A}_{2}+B \tilde{K}_{1}\right) x+\left(\tilde{E}_{2}+B \tilde{K}_{2}\right) \tilde{p}_{2}+\right.$ $\left.E_{w} w\right]+\alpha_{2} x^{\top} P_{3} x-\mu_{2}\|w\|^{2} \leq 0$. Let $V_{2}(x)=x^{\top} P_{3} x$. Then, we have $\dot{V}_{2}=2 x^{\top} P_{3}\left[\left(\tilde{A}_{2}+B \tilde{K}_{1}\right) x+\left(\tilde{E}_{2}+B \tilde{K}_{2}\right) \tilde{p}_{2}-B \Delta k+\right.$ $\left.E_{w} w\right] \leq-\alpha_{2} x^{\top} P_{3} x+\mu_{2}\|w\|^{2}+2\left\|P_{3} B\right\|\|x\|\|\Delta k\|$. Recalling $\|\Delta k\| \leq \hat{\kappa}\|e\|$, we have

$$
\dot{V}_{2} \leq-\alpha_{2} x^{\top} P_{3} x+\mu_{2}\|w\|^{2}+\theta\|x\|\|e\|
$$

where $\theta=2\left\|P_{3} B\right\| \hat{\kappa}$. The rest of the proof proceeds as that given in part 5) of the proof of Theorem 1 .

Remark 7. Inequality (61) is an LMI in decision variables $\mu_{1}, P_{1}, R_{1}, R_{2}$ when $\alpha_{1}$ is fixed, (62) is an LMI in decision variables $\mu_{2}, P_{2}, R_{3}, R_{4}$ when $\alpha_{2}$ and $X_{2}$ are fixed, and (63) is an LMI in decision variables $X_{1}, Y_{1}$. Hence, we can fix $\alpha_{1}, \alpha_{2}, X_{2}$ and solve for (61)-63). When $L_{1}, L_{2}, K_{1}, K_{2}$ are obtained, a re-computation for $P, \alpha_{0}, \mu$ using Proposition 2 may result in a better convergence rate guarantee.

Remark 8. The LMIs (29) and (30) both have dimensions $\left(n_{x}+n_{p}+n_{q}+n_{w}\right) \times\left(n_{x}+n_{p}+n_{q}+n_{w}\right)$, the LMIs (61) and (62) both have dimensions $\left(n_{x}+n_{p}+n_{w}\right) \times\left(n_{x}+n_{p}+n_{w}\right)$, and the LMI 63) has dimension $n_{p} \times n_{p}$. These LMIs can be solved reliably and efficiently by the interior point method algorithms of convex optimization with a polynomial-time complexity. Exploring for what class of systems these LMIs are guaranteed to be feasible (i.e., analytical verification of feasibility) is still under our investigation. Furthermore, these LMI conditions might be conservative compared with specific results that focus on certain special nonlinearities such as the globally Lipschitz nonlinearity.

\section{EVEnt-TRIGgered Control Design}

In this section, we discuss event-triggering mechanisms (ETMs) within the observer-based controller designed in the preceding section for the system described by (11)-(2) where the nonlinearity $p$ is assumed to be globally Lipschitz. For certain incrementally quadratic nonlinearities that imply the global Lipschitzness (such as the incremental sector bounded nonlinearity and the nonlinearities with Jacobians in polytopes [48]), using their corresponding incremental matrix characterizations, instead of the matrix characterizations for global Lipschitzness, makes the associated LMIs in the design procedure less conservative, while benefiting from having the Lipschitz property needed for the upcoming ETM-related results to hold.

\section{A. Configuration I: The Controller Channel Is Implemented By ETM}

In this subsection, we discuss the configuration shown in Figure 1 where the plant is described by (1)-(2), the observer is given in 11, the continuous-time feedback controller is 


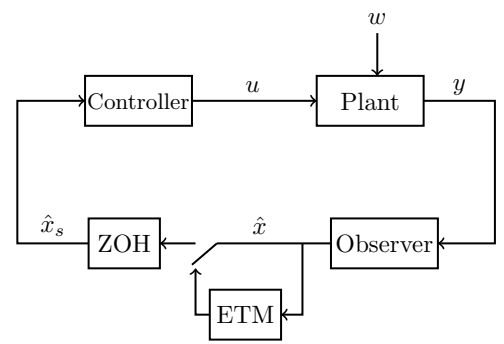

Fig. 1. Configuration where the ETM is implemented in the controller channel.

given in (12), and the ETM only has the information of $\hat{x}$, the state of the observer. We will assume that $\|w\|_{\infty} \leq \omega_{0}$ where $\omega_{0}$ is a positive constant indicating the bound of the disturbance in this subsection and the next subsection.

The feedback controller $u(t)$ is implemented by an ETM such that it is only updated at certain triggering time instances $t_{1}, t_{2}, \ldots$ where $t_{k}<t_{k+1}$ for any $k \geq 0$ and kept constant during consecutive time instances. Define $t_{0}=0$ and the piecewise constant signal $\hat{x}_{s}$ as

$$
\hat{x}_{s}(t)=\hat{x}\left(t_{k}\right), \forall t \in\left[t_{k}, t_{k+1}\right) .
$$

Then the control input $u(t)$ is given by

$$
u(t)=K_{1} \hat{x}_{s}(t)+K_{2} p\left(C_{q} \hat{x}_{s}(t)\right)
$$

where $K_{1}, K_{2}$ are matrices to be designed. The input $u(t)$ has the same form as that in (12), but it is updated at triggering time instances $t_{1}, t_{2}, \ldots$, which are determined by the following type of triggering rule:

$$
t_{k+1}=\inf \left\{t \mid t \geq t_{k}+\tau,\left\|\hat{x}_{e}(t)\right\|>\sigma\|\hat{x}(t)\|+\epsilon\right\}
$$

where $\hat{x}_{e}$ is defined as $\hat{x}_{e}(t)=\hat{x}_{s}(t)-\hat{x}(t)$ and $\tau, \sigma, \epsilon$ are all positive numbers to be specified. The time-updating rule $(72)$ guarantees that the inter-execution times $\left\{t_{k+1}-t_{k}\right\}$ are lower bounded by the built-in positive constant $\tau$, which means that Zeno phenomenon (i.e., infinite executions happen in a finite amount of time) will not occur [55].

Remark 9. The triggering rule (72) only depends on the information of $\hat{x}$ and $\hat{x}_{e}$, which are available from the proposed observer. This triggering rule is a combination of a mixed ETM and a time regularization technique. There are several motivations for choosing this type of rule. It was known that even in the absence of disturbances, inter-execution times of many ETMs converge to zero for output-based control configurations [59]. To exclude the Zeno phenomenon, time regularization or periodic event-triggered control, which enforces a builtin lower bound for inter-execution times, has been utilized in recent works on observer-based ETMs [62], [57], [61]. Furthermore, mixed ETM is known to be robust to external disturbances or measurement noise, while relative ETM and absolute ETM have zero robustness to disturbance/noise [60]. Additionally, an event-triggering rule with time regularization can benefit from using mixed ETMs in terms of the number of events that are generated (e.g., see Example 3 in [60]).

The closed-loop system that combines system (1)-(2), ob- server (11) and event-triggered controller (71) is expressed compactly as

$$
\dot{z}=A_{c} z+H_{1} p+H_{2} \delta p+H_{3} \delta \hat{p}+H_{4} w+H_{5} \hat{x}_{e}
$$

where $\delta p, \delta q$ are given in (16), $A_{c}$ is given in (18), $H_{1}, H_{2}, H_{3}, H_{4}$ are given in (19), and

$$
\begin{aligned}
\delta \hat{p} & =p\left(C_{q} \hat{x}_{s}\right)-p\left(C_{q} x\right), \\
H_{5} & =\left(\begin{array}{c}
B K_{1} \\
\mathbf{0}
\end{array}\right) .
\end{aligned}
$$

Theorem 3. Consider the configuration shown in Figure 1 where the plant is described by (1)-(2) with $p(\mathbf{0})=\mathbf{0}$ and $\|w\|_{\infty} \leq \omega_{0}$ with $\omega_{0}$ a positive number. Suppose that there exists $\ell>0$ such that $\|p(r)-p(s)\| \leq \ell\|r-s\|$ for any $r, s$. Suppose that there exist positive numbers $\alpha_{0}>0, \mu>0$, and matrices $P \succ 0, K_{1}, K_{2}, L_{1}, L_{2}$ such that the closed-loop system (17) with controller (12) and observer (11) satisfies $\dot{V} \leq-\alpha_{0} V+\mu\|w\|^{2}$ where $V=z^{\top} P z$. Choose any $\epsilon>0$ and

$$
\sigma=\frac{\varrho \alpha_{0} \lambda_{m}(P)}{2 \sqrt{2} s}>0
$$

where $0<\varrho<1$ and $s=\left\|P H_{5}\right\|+\ell\left\|P H_{3}\right\|\left\|C_{q}\right\|$. Choose $\tau>0$ as the solution to the equation $\phi(\tau)=1$ where $\phi$ is the solution of the following ODE:

$$
\dot{\phi}=\sqrt{2}\left(\eta_{4}+\eta_{2} \phi\right)(1+\sigma \phi), \phi(0)=0,
$$

with

$$
\left\{\begin{array}{l}
\eta_{1}=\left\|A_{c}\right\|+\ell \sqrt{b_{1}^{2}+b_{2}^{2}}, \\
\eta_{2}=\left\|H_{5}\right\|+\ell\left\|H_{3}\right\|\left\|C_{q}\right\|, \\
\eta_{3}=\ell\left\|H_{2}\right\|\left\|L_{1} F_{w}\right\|+\left\|H_{4}\right\|, \\
\eta_{4}=\frac{\eta_{1}}{\sqrt{2} \sigma}+\frac{\eta_{3} \omega_{0}}{\epsilon}, \\
b_{1}=\left\|H_{1}\right\|\left\|C_{q}\right\|, \\
b_{2}=\left\|H_{2}\right\|\left\|C_{q}+L_{1} C\right\|+\left\|H_{3}\right\|\left\|C_{q}\right\| .
\end{array}\right.
$$

Then, the closed-loop system (73) that implements the triggering rule $\sqrt{72}$ is ISpS w.r.t. w.

Proof. Since the derivative of $V$ along the trajectory of the closed-loop system (17) satisfies $\dot{V} \leq-\alpha_{0} V+\mu\|w\|^{2}$, the derivative of $V$ along the trajectory of the closed-loop system (73) satisfies $\dot{V} \leq-\alpha_{0} V+\mu\|w\|^{2}+2 z^{\top} P\left[H_{5} \hat{x}_{e}+H_{3}(\delta \hat{p}-\right.$ $\Delta p)] \leq-\alpha_{0} \lambda_{m}(P)\|z\|^{2}+\mu\|w\|^{2}+2\|z\|\left(\left\|P H_{5}\right\|\left\|\hat{x}_{e}\right\|+\right.$ $\left.\left\|P H_{3}\right\|\|\delta \hat{p}-\Delta p\|\right)$. Clearly, $\|\delta \hat{p}-\Delta p\|=\| p\left(C_{q} \hat{x}_{s}\right)-$ $p\left(C_{q} \hat{x}\right)\|\leq \ell\| C_{q}\left(\hat{x}_{s}-\hat{x}\right)\|\leq \ell\| C_{q}\|\| \hat{x}_{e} \|$. Then, we have

$$
\begin{aligned}
\dot{V} \leq- & \alpha_{0} \lambda_{m}(P)\|z\|^{2}+\mu\|w\|^{2}+2 s\|z\|\left\|\hat{x}_{e}\right\| \\
\leq- & (1-\varrho) \alpha_{0} \lambda_{m}(P)\|z\|^{2}+\mu\|w\|^{2} \\
& +\|z\|\left[2 s\left\|\hat{x}_{e}\right\|-\varrho \alpha_{0} \lambda_{m}(P)\|z\|\right] .
\end{aligned}
$$

For any $x, e$, we have $\|z\|=\sqrt{\|x\|^{2}+\|x-\hat{x}\|^{2}}=$ $\sqrt{\|\hat{x}\|^{2}+2\|x\|^{2}-2 x^{\top} \hat{x}} \geq\|\hat{x}\| / \sqrt{2}$, meaning that $\|\hat{x}\| \leq$ $\sqrt{2}\|z\|$. Therefore, the condition

$$
\left\|\hat{x}_{e}\right\| \leq \sigma\|\hat{x}\|+\epsilon
$$


implies

$$
\left\|\hat{x}_{e}\right\| \leq \sqrt{2} \sigma\|z\|+\epsilon,
$$

which is equivalent to the inequality $2 s\left\|\hat{x}_{e}\right\|$ $\varrho \alpha_{0} \lambda_{m}(P)\|z\| \leq 2 s \epsilon$.

Choose a constant $c$ such that $0<c<(1-\varrho) \alpha_{0} \lambda_{m}(P)$. Then, as long as (80) holds, from (78) we have

$$
\dot{V} \leq-\left[(1-\varrho) \alpha_{0} \lambda_{m}(P)-c\right]\|z\|^{2}+\mu\|w\|^{2}+\frac{s^{2} \epsilon^{2}}{c} .
$$

Recalling that $p(\mathbf{0})=\mathbf{0}$ and $\ell$ is the Lipschitz constant of $p$, we have $\|p\| \leq \ell\left\|C_{q}\right\|\|x\|,\|\delta p\| \leq \ell\|\delta q\| \leq \ell\left(\| C_{q}+\right.$ $\left.L_{1} C\|\| e\|+\| L_{1} F_{w}\|\| w \|\right)$, and $\|\delta \hat{p}\| \leq \ell\left\|C_{q}\right\|\left(\left\|\hat{x}_{e}\right\|+\|e\|\right)$. Therefore, from 73 we have

$$
\begin{gathered}
\|\dot{z}\| \leq\left\|A_{c}\right\|\|z\|+\left\|H_{5}\right\|\left\|\hat{x}_{e}\right\|+\ell\left\|H_{1}\right\|\left\|C_{q}\right\|\|x\| \\
+\ell\left\|H_{2}\right\|\left(\left\|C_{q}+L_{1} C\right\|\|e\|+\left\|L_{1} F_{w}\right\|\|w\|\right) \\
\quad+\ell\left\|H_{3}\right\|\left\|C_{q}\right\|\left(\left\|\hat{x}_{e}\right\|+\|e\|\right)+\left\|H_{4}\right\|\|w\| \\
\leq \eta_{1}\|z\|+\eta_{2}\left\|\hat{x}_{e}\right\|+\eta_{3}\|w\|
\end{gathered}
$$

where the second inequality follows from Cauchy's inequality $b_{1}\|x\|+b_{2}\|e\| \leq \sqrt{b_{1}^{2}+b_{2}^{2}}\|z\|$.

Because $\|\dot{z}\|=\sqrt{\|\dot{x}\|^{2}+\|\dot{e}\|^{2}}=\sqrt{\|\dot{x}\|^{2}+\|\dot{x}-\dot{\hat{x}}\|^{2}}=$ $\sqrt{\|\dot{\hat{x}}\|^{2}+2\|\dot{x}\|^{2}-2 \dot{x}^{\top} \dot{\hat{x}}} \geq\|\dot{\hat{x}}\| / \sqrt{2}$ and $\left\|\dot{\hat{x}}_{e}\right\|=\|\dot{\hat{x}}\|$, we have $\left\|\dot{\hat{x}}_{e}\right\| \leq \sqrt{2}\|\dot{z}\|$.

Let $v(t)=\frac{\left\|\hat{x}_{e}(t)\right\|}{\sqrt{2} \sigma\|z(t)\|+\epsilon}$. Then for any $h>0$,

$$
\begin{aligned}
& v(t+h)-v(t)=\frac{\left\|\hat{x}_{e}(t+h)\right\|}{\sqrt{2} \sigma\|z(t+h)\|+\epsilon}-\frac{\left\|\hat{x}_{e}(t)\right\|}{\sqrt{2} \sigma\|z(t)\|+\epsilon} \\
& =\frac{\left\|\hat{x}_{e}(t+h)\right\|(\sqrt{2} \sigma\|z(t)\|+\epsilon)-\left\|\hat{x}_{e}(t)\right\|(\sqrt{2} \sigma\|z(t+h)\|+\epsilon)}{(\sqrt{2} \sigma\|z(t+h)\|+\epsilon)(\sqrt{2} \sigma\|z(t)\|+\epsilon)} \\
& =\frac{\left(\left\|\hat{x}_{e}(t+h)-\right\| \hat{x}_{e}(t) \|\right)(\sqrt{2} \sigma\|z(t)\|+\epsilon)}{(\sqrt{2} \sigma\|z(t+h)\|+\epsilon)(\sqrt{2} \sigma\|z(t)\|+\epsilon)} \\
& -\frac{\sqrt{2} \sigma\left\|\hat{x}_{e}(t)\right\|(\|z(t+h)\|-\|z(t)\|)}{(\sqrt{2} \sigma\|z(t+h)\|+\epsilon)(\sqrt{2} \sigma\|z(t)\|+\epsilon)}
\end{aligned}
$$

and hence

$$
\begin{aligned}
D^{+} v(t) & =\lim \sup _{h \rightarrow 0^{+}} \frac{v(t+h)-v(t)}{h} \\
& =\frac{D^{+}\left\|\hat{x}_{e}(t)\right\|}{\sqrt{2} \sigma\|z(t)\|+\epsilon}-\frac{\sqrt{2} \sigma\left\|\hat{x}_{e}(t)\right\| D^{+}\|z(t)\|}{(\sqrt{2} \sigma\|z(t)\|+\epsilon)^{2}} .
\end{aligned}
$$

When $z(t) \neq 0, D^{+}\|z(t)\|=\frac{z(t)^{T} \dot{z}(t)}{\|z(t)\|}$ and therefore $\left|D^{+}\|z(t)\|\right| \leq\|\dot{z}(t)\|$. When $z(t)=0, D^{+}\|z(t)\|=$ $\limsup _{h \rightarrow 0^{+}} \frac{\|z(t+h)\|-\|z(t)\|}{h}=\limsup _{h \rightarrow 0^{+}}\left\|\frac{z(t+h)}{h}\right\|=$ $\|\dot{z}(t)\|$. Thus, in all cases $\left|D^{+}\|z(t)\|\right| \leq\|\dot{z}(t)\|$. Similarly, $\left|D^{+}\left\|\hat{x}_{e}(t)\right\|\right| \leq\left\|\dot{\hat{x}}_{e}(t)\right\|$. Dropping the argument $t$, it now follows from $(82)$ that

$$
\begin{aligned}
D^{+} v & \leq \frac{\left\|\dot{\hat{x}}_{e}\right\|}{\sqrt{2} \sigma\|z\|+\epsilon}+\frac{\sqrt{2} \sigma\left\|\hat{x}_{e}\right\|\|\dot{z}\|}{(\sqrt{2} \sigma\|z\|+\epsilon)^{2}} \\
& \leq \frac{\sqrt{2}\|\dot{z}\|}{\sqrt{2} \sigma\|z\|+\epsilon}+\frac{\sqrt{2} \sigma\left\|\hat{x}_{e}\right\|\|\dot{z}\|}{(\sqrt{2} \sigma\|z\|+\epsilon)^{2}} \\
& =\frac{\sqrt{2}\|\dot{z}\|}{\sqrt{2} \sigma\|z\|+\epsilon}\left(1+\frac{\sigma\left\|\hat{x}_{e}\right\|}{\sqrt{2} \sigma\|z\|+\epsilon}\right)
\end{aligned}
$$

$$
\begin{aligned}
& \leq \sqrt{2}\left(\eta_{4}+\eta_{2} \frac{\left\|\hat{x}_{e}\right\|}{\sqrt{2} \sigma\|z\|+\epsilon}\right)\left(1+\sigma \frac{\left\|\hat{x}_{e}\right\|}{\sqrt{2} \sigma\|z\|+\epsilon}\right) \\
& =\sqrt{2}\left(\eta_{4}+\eta_{2} v\right)(1+\sigma v)
\end{aligned}
$$

where the following facts are used to derive the last inequality:

$$
\frac{\eta_{1}\|z\|}{\sqrt{2} \sigma\|z\|+\epsilon} \leq \frac{\eta_{1}}{\sqrt{2} \sigma}, \frac{\eta_{3}\|w\|}{\sqrt{2} \sigma\|z\|+\epsilon} \leq \frac{\eta_{3} \omega_{0}}{\epsilon} .
$$

Since $v\left(t_{k}\right)=0$, it now follows from the comparison lemma that $v(t) \leq \phi\left(t-t_{k}\right)$. Since the time it takes for $v$ to evolve from 0 to 1 is lower bounded by $\tau$, 80 holds during the time interval $\left[t_{k}, t_{k}+\tau\right]$. For any $k \geq 0$, if $t_{k+1}=t_{k}+\tau$, then 80 holds during the interval $\left[t_{k} \cdot t_{k+1}\right)$ as shown above; if $t_{k+1}>t_{k}+\tau$, then, during the interval $\left[t_{k}+\tau, t_{k+1}\right)$, condition (79) holds, which implies that 800 holds. Therefore, 80 holds during any interval $\left[t_{k}, t_{k+1}\right)$ for any $k \geq 0$, i.e., it holds for any $t \geq 0$. Since satisfaction of 80 implies the inequality (81), we conclude that the function $V$ is an ISpS-Lyapunov function since it satisfies (8) for any $t \geq 0$ with $\gamma(\|z\|)=$ $\left[(1-\varrho) \alpha_{0} \lambda_{m}(P)-c\right]\|z\|^{2} \in \mathcal{K}_{\infty}, \chi(\|w\|)=\mu\|w\|^{2} \in \mathcal{K}$ and $d=s^{2} \epsilon^{2} / c>0$. The conclusion follows by Proposition 1.

Remark 10. In the proof of Theorem 3 the equation for $\tau$ is given explicitly, and any $\tau^{\prime} \in(0, \tau]$ also makes the proof valid. The parameter $\epsilon$ can be chosen arbitrarily, but there are trade-offs in choosing $\epsilon$ : on one hand, the value of $d$ in the inequality (8) or (10) increases as $\epsilon$ increases, meaning that the ultimate bound for $x$ increases as $\epsilon$ increases; on the other hand, the explicit equation of $\tau$ depends on $\epsilon$, with $\tau$ decreasing to 0 when $\epsilon$ approaches 0 . Hence, parameters in the triggering rule should be chosen appropriately to balance the execution times and the performance. Finding the maximal lower-bound of the inter-execution times is an interesting and challenging problem that will be investigated in our future work.

\section{B. Configuration II: The Controller and Observer Channels Are Both Implemented By ETMs}

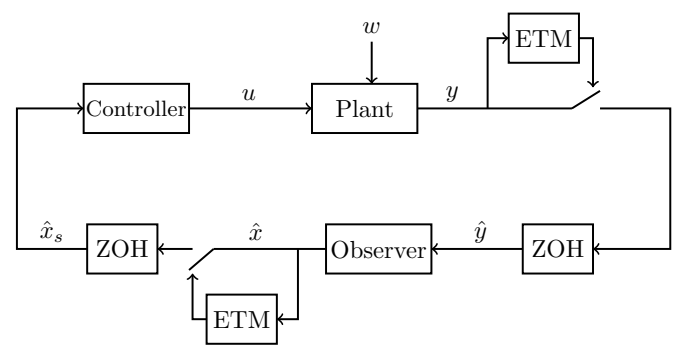

Fig. 2. Configuration where ETMs are implemented in both the observer and controller channels asynchronously

In this subsection, we discuss the configuration shown in Figure 2 where the ETM for the output is triggered by the information of $y$ and the ETM for the input is triggered by the information of $\hat{x}$, in an asynchronous manner.

Consider a system described by (1)-2). The observer in the configuration of Figure 2 only has sampled information $y_{s}(t)$ 
of the output $y(t)$ where $y_{s}(t)$ is updated at time instances $t_{1}^{y}, t_{2}^{y}, \ldots$ by

$$
y_{s}(t)=y\left(t_{k}^{y}\right), \forall t \in\left[t_{k}^{y}, t_{k+1}^{y}\right) .
$$

Here, $t_{0}^{y}=0$ and the triggering times $t_{1}^{y}, t_{2}^{y}, \ldots$ are determined by the following triggering rule:

$$
t_{k+1}^{y}=\inf \left\{t \mid t \geq t_{k}^{y}+\tau_{y},\left\|y_{e}(t)\right\|>\sigma_{y}\|y(t)\|+\epsilon_{y}\right\}
$$

where $y_{e}(t)=y_{s}(t)-y(t)$ and $\tau_{y}, \sigma_{y}, \epsilon_{y}$ are all positive numbers to be specified.

With the sampled information $y_{s}(t)$, the observer now becomes

$$
\left\{\begin{array}{l}
\dot{\hat{x}}=A \hat{x}+B u+E_{p} p\left(\hat{q}+L_{1}\left(\hat{y}-y_{s}\right)\right)+L_{2}\left(\hat{y}-y_{s}\right), \\
\hat{y}=C \hat{x}+D u, \\
\hat{q}=C_{q} \hat{x},
\end{array}\right.
$$

where $L_{1}, L_{2}$ are matrices to be designed.

The observer-based controller $u(t)$ has the form shown in (71) where $\hat{x}_{s}(t)$ is updated at time instances $t_{1}^{u}, t_{2}^{u}, \ldots$ by

$$
\hat{x}_{s}(t)=\hat{x}\left(t_{k}^{u}\right), \forall t \in\left[t_{k}^{u}, t_{k+1}^{u}\right) .
$$

Here, $t_{0}^{u}=0$ and the triggering times $t_{1}^{u}, t_{2}^{u}, \ldots$ are determined by the following triggering rule:

$$
t_{k+1}^{u}=\inf \left\{t \mid t \geq t_{k}^{u}+\tau_{u},\left\|\hat{x}_{e}(t)\right\|>\sigma_{u}\|\hat{x}(t)\|+\epsilon_{u}\right\}
$$

where $\hat{x}_{e}(t)=\hat{x}\left(t_{k}\right)-\hat{x}(t)$ and $\tau_{u}, \sigma_{u}, \epsilon_{u}$ are all positive numbers to be specified. Note that the information of $\hat{x}$ and $\hat{x}_{e}$ are available from the proposed observer.

The time-updating rule (83) (or 85) provides a built-in positive lower bound $\tau_{y}$ (or $\tau_{u}$ ) for inter-execution times $\left\{t_{k+1}^{y}-t_{k}^{y}\right\}$ (or $\left\{t_{k+1}^{u}-t_{k}^{u}\right\}$ ), implying that the Zeno phenomenon will not occur. Although there is no bound guarantee on the inter-execution times between $t_{k}^{y}$ and $t_{k}^{u}$, this will not cause a problem since these two ETMs are implemented separately.

Since $y_{e}(t)=y_{s}(t)-y(t)$, the closed-loop system that combines system (1)-(2), observer (84) and event-triggered controller (71) is expressed compactly as

$$
\dot{z}=A_{c} z+H_{1} p+H_{2} \delta \tilde{p}+H_{3} \delta \hat{p}+H_{4} w+H_{5} \hat{x}_{e}+H_{6} y_{e}
$$

where $A_{c}, H_{1}, H_{2}, H_{3}, H_{4}, H_{5}$ are given in (18), 19, , 75, respectively, $\delta \hat{p}$ is given in (74, , and

$$
\begin{aligned}
\delta \tilde{p} & =p(q+\delta \tilde{q})-p(q) \\
\delta \tilde{q} & =-\left(C_{q}+L_{1} C\right) e-L_{1} F_{w} w-L_{1} y_{e} \\
H_{6} & =\left(\begin{array}{c}
\mathbf{0} \\
L_{2}
\end{array}\right) .
\end{aligned}
$$

Theorem 4. Consider the configuration shown in Figure 2 where the plant is described by (1)-(2) with $D=\mathbf{0}, p(\mathbf{0})=\mathbf{0}$, and $\|w\|_{\infty} \leq \omega_{0}$ with $\omega_{0}$ a positive number. Suppose that there exists $\ell>0$ such that $\|p(r)-p(s)\| \leq \ell\|r-s\|$ for any $r, s$. Suppose that there exist constants $\alpha_{0}>0, \mu>0$, and matrices $P \succ 0, K_{1}, K_{2}, L_{1}, L_{2}$ such that the closed-loop system (17) with controller (12) and observer (11) satisfies $\dot{V} \leq-\alpha_{0} V+\mu\|w\|^{2}$ where $V=z^{\top} P z$. Choose any $\epsilon_{y}, \epsilon_{u}>$
0 , and

$$
\sigma_{y}=\frac{a_{2} \varrho \alpha_{0} \lambda_{m}(P)}{2\|C\| s_{2}}, \quad \sigma_{u}=\frac{a_{1} \varrho \alpha_{0} \lambda_{m}(P)}{2 \sqrt{2} s_{1}},
$$

where $0<\varrho<1, s_{1}=\left\|P H_{5}\right\|+\ell\left\|P H_{3}\right\|\left\|C_{q}\right\|, s_{2}=$ $\left\|P H_{6}\right\|+\ell\left\|P H_{2}\right\|\left\|L_{1}\right\|$, and $a_{1}, a_{2}$ are two constants satisfying $0<a_{1}, a_{2}<1$ and $a_{1}+a_{2}=1$. Choose $\tau_{u}>0$ as the solution to the equation $\phi_{1}\left(\tau_{u}\right)=1$ where $\phi_{1}$ is the solution of the following $O D E$

$$
\dot{\phi}_{1}=\sqrt{2}\left(1+\sigma_{u} \phi_{1}\right)\left(\eta_{5}+\eta_{2} \phi_{1}+d_{1} \eta_{7}\right), \phi_{1}(0)=0
$$

and choose $\tau_{y}>0$ as the solution to the equation $\phi_{2}\left(\tau_{y}\right)=1$ where $\phi_{2}$ is the solution of the following $O D E$

$$
\dot{\phi}_{2}=\|C\|\left(1+\sigma_{y} \phi_{2}\right)\left(\eta_{6}+\eta_{7} \phi_{2}+d_{2} \eta_{2}\right), \phi_{2}(0)=0
$$

where

$$
\left\{\begin{array}{l}
\eta_{5}=\frac{\eta_{1}}{\sqrt{2} \sigma_{u}}+\frac{\eta_{2} \omega_{0}}{\epsilon_{u}}, \\
\eta_{6}=\frac{\eta_{1}}{\sigma_{y}\|C\|}+\frac{\eta_{3} \omega_{0}}{\epsilon_{y}}, \\
\eta_{7}=\ell\left\|H_{2}\right\|\left\|L_{1}\right\|+\left\|H_{6}\right\|, \\
d_{1}=\max \left\{\frac{\epsilon_{y}}{\epsilon_{u}}, \frac{\sigma_{y}\|C\|}{\sqrt{2} \sigma_{u}}\right\}, \\
d_{2}=\max \left\{\frac{\epsilon_{u}}{\epsilon_{y}}, \frac{\sqrt{2} \sigma_{u}}{\sigma_{y}\|C\|}\right\},
\end{array}\right.
$$

and $\eta_{1}, \eta_{2}, \eta_{3}$ are given in (77). Then, the closed-loop system (86) that impllements triggering rules (83) and (85) is ISpS w.r.t. $w$.

Proof. If the derivative of $V$ along the trajectory of (17) satisfies $\dot{V} \leq-\alpha_{0} V+\mu\|w\|^{2}$, then the derivative of $V$ along the trajectory of the closed-loop system satisfies

$$
\begin{aligned}
\dot{V} \leq & -\alpha_{0} V+\mu\|w\|^{2}+2 z^{\top} P\left[H_{2}(\delta \tilde{p}-\delta p)\right. \\
& \left.+H_{3}(\delta \hat{p}-\Delta p)+H_{5} \hat{x}_{e}+H_{6} y_{e}\right] \\
\leq & -(1-\varrho) \alpha_{0} \lambda_{m}(P)\|z\|^{2}+\mu\|w\|^{2}+\|z\|\left(2 s_{1}\left\|\hat{x}_{e}\right\|\right. \\
& \left.+2 s_{2}\left\|y_{e}\right\|-\varrho \alpha_{0} \lambda_{m}(P)\|z\|\right)
\end{aligned}
$$

where the following facts are used: $\|\delta \tilde{p}-\delta p\| \leq \ell\|\delta \tilde{q}-\delta q\| \leq$ $\ell\left\|L_{1}\right\|\left\|y_{e}\right\|,\|\delta \hat{p}-\Delta p\| \leq \ell\left\|C_{q}\right\|\left\|\hat{x}_{e}\right\|$. As $\|z\| \geq\|\hat{x}\| / \sqrt{2}$ and $\|z\| \geq\|x\| \geq\|y\| /\|C\|$, we have

$$
\|z\| \geq \frac{a_{1}\|\hat{x}\|}{\sqrt{2}}+\frac{a_{2}\|y\|}{\|C\|} .
$$

From 89 and 90 we have

$$
\begin{aligned}
& \dot{V} \leq-(1-\varrho) \alpha_{0} \lambda_{m}(P)\|z\|^{2}+\mu\|w\|^{2}+\|z\|\left[2 s_{1}\left\|\hat{x}_{e}\right\|\right. \\
& \left.-\frac{a_{1} \varrho \alpha_{0} \lambda_{m}(P)}{\sqrt{2}}\|\hat{x}\|\right]+\|z\|\left[2 s_{2}\left\|y_{e}\right\|-\frac{a_{2} \varrho \alpha_{0} \lambda_{m}(P)}{\|C\|}\|y\|\right] .
\end{aligned}
$$

The condition $\left\|\hat{x}_{e}\right\| \leq \sigma_{u}\|\hat{x}\|+\epsilon_{u}$ implies

$$
\left\|\hat{x}_{e}\right\| \leq \sqrt{2} \sigma_{u}\|z\|+\epsilon_{u}
$$

and the condition $\left\|y_{e}\right\| \leq \sigma_{y}\|y\|+\epsilon_{y}$ implies

$$
\left\|y_{e}\right\| \leq \sigma_{y}\|C\|\|z\|+\epsilon_{y} .
$$


As long as 91 and 92 hold, we have

$$
\dot{V} \leq-\left[(1-\varrho) \alpha_{0} \lambda_{m}(P)-c\right]\|z\|^{2}+\mu\|w\|^{2}+\frac{\epsilon_{0}^{2}}{4 c}
$$

where $\epsilon_{0}=2\left(s_{1} \epsilon_{u}+s_{2} \epsilon_{y}\right)$, and $c$ is a constant satisfying $0<c<(1-\varrho) \alpha_{0} \lambda_{m}(P)$.

Since $\|p\| \leq \ell\left\|C_{q}\right\|\|x\|,\|\delta \hat{p}\| \leq \ell\left\|C_{q}\right\|\left(\left\|\hat{x}_{e}\right\|+\|e\|\right)$, and $\|\delta \tilde{p}\| \leq \ell\left(\left\|C_{q}+L_{1} C\right\|\|e\|+\left\|L_{1} F_{w}\right\|\|w\|+\left\|L_{1}\right\|\left\|y_{e}\right\|\right)$, from (86) we have $\|\dot{z}\| \leq \eta_{1}\|z\|+\eta_{2}\left\|\hat{x}_{e}\right\|+\eta_{3}\|w\|+\eta_{7}\left\|y_{e}\right\|$.

Similar to the argument in the proof of Theorem 3, we can show the following inequality holds when $\left\|\hat{x}_{e}\right\| \neq 0$ and $\|z\| \neq 0$ :

$$
\begin{aligned}
& \frac{\mathrm{d}}{\mathrm{d} t}\left(\frac{\left\|\hat{x}_{e}\right\|}{\sqrt{2} \sigma_{u}\|z\|+\epsilon_{u}}\right) \leq \sqrt{2}\left(1+\frac{\sigma_{u}\left\|\hat{x}_{e}\right\|}{\sqrt{2} \sigma_{u}\|z\|+\epsilon_{u}}\right) \times \\
& \left(\eta_{5}+\frac{\eta_{2}\left\|\hat{x}_{e}\right\|}{\sqrt{2} \sigma_{u}\|z\|+\epsilon_{u}}+\frac{\eta_{7}\left\|y_{e}\right\|}{\sqrt{2} \sigma_{u}\|z\|+\epsilon_{u}}\right) .
\end{aligned}
$$

It is easy to verify that $\frac{\eta_{7}\left\|y_{e}\right\|}{\sqrt{2} \sigma_{u}\|z\|+\epsilon_{u}} \leq d_{1} \frac{\eta_{7}\left\|y_{e}\right\|}{\sigma_{y}\|C\|\|z\|+\epsilon_{y}}$. Hence, from 94 we have

$$
\begin{aligned}
& \frac{\mathrm{d}}{\mathrm{d} t}\left(\frac{\left\|\hat{x}_{e}\right\|}{\sqrt{2} \sigma_{u}\|z\|+\epsilon_{u}}\right) \leq \sqrt{2}\left(1+\frac{\sigma_{u}\left\|\hat{x}_{e}\right\|}{\sqrt{2} \sigma_{u}\|z\|+\epsilon_{u}}\right) \times \\
& \left(\eta_{5}+\frac{\eta_{2}\left\|\hat{x}_{e}\right\|}{\sqrt{2} \sigma_{u}\|z\|+\epsilon_{u}}+d_{1} \frac{\eta_{7}\left\|y_{e}\right\|}{\sigma_{y}\|C\|\|z\|+\epsilon_{y}}\right) .
\end{aligned}
$$

When $\left\|\hat{x}_{e}\right\|=0$ or $\|z\|=0$, the upper right-hand derivative of $\frac{\left\|\hat{x}_{e}\right\|}{\overline{2} \sigma_{u}\|z\|+\epsilon_{u}}$ can be calculated similar to the proof of Theorem 3. which can still be captured by the inequality above.

Since $\left\|\dot{y}_{e}\right\|=\|\dot{y}\| \leq\|C\|\|\dot{x}\| \leq\|C\|\|\dot{z}\|$, we can show that the following inequality holds using arguments similar to those used above:

$$
\begin{aligned}
& \frac{\mathrm{d}}{\mathrm{d} t}\left(\frac{\left\|y_{e}\right\|}{\sigma_{y}\|C\|\|z\|+\epsilon_{y}}\right) \leq\|C\|\left(1+\frac{\sigma_{y}\left\|y_{e}\right\|}{\sigma_{y}\|C\|\|z\|+\epsilon_{y}}\right) \times \\
& \left(\eta_{6}+\frac{\eta_{7}\left\|y_{e}\right\|}{\sigma_{y}\|C\|\|z\|+\epsilon_{y}}+d_{2} \frac{\eta_{2}\left\|\hat{x}_{e}\right\|}{\sqrt{2} \sigma_{u}\|z\|+\epsilon_{u}}\right)
\end{aligned}
$$

where the discussion on using the upper right-hand derivative is omitted since it is similar to that used in the proof of Theorem 3 .

It is not hard to show that the time it takes for $\left\|\hat{x}_{e}\right\|$ (resp. $\left\|y_{e}\right\|$ ) to evolve from 0 to $\sqrt{2} \sigma_{u}\|z\|+\epsilon_{u}$ (resp. $\sigma_{y}\|C\|\|z\|+\epsilon_{y}$ ) is lower bounded by $\tau_{u}$ (resp. $\tau_{y}$ ), which implies that (91) holds during $\left[t_{k}^{u}, t_{k}^{u}+\tau_{u}\right)$, and (92) holds during $\left[t_{k}^{y}, t_{k}^{y}+\tau_{y}\right)$, for any $k \geq 0$. Recalling that $\left\|\hat{x}_{e}\right\| \leq \sigma_{u}\|\hat{x}\|+\epsilon_{u}$ implies (91) and $\left\|y_{e}\right\| \leq \sigma_{y}\|y\|+\epsilon_{y}$ implies (92), the triggering rules (83) and (85) guarantee that (91) holds during the interval $\left[t_{k}^{u}, t_{k+1}^{u}\right)$ for any $k \geq 0$, and (92) holds during the interval $\left[t_{k}^{y}, t_{k+1}^{y}\right)$ for any $k \geq 0$. Hence, (93) holds for any $t \geq 0$, implying that the function $V$ is an ISpS-Lyapunov function since it satisfies (8) with $\gamma(\|z\|)=\left[(1-\varrho) \alpha_{0} \lambda_{m}(P)-c\right]\|z\|^{2} \in \mathcal{K}_{\infty}, \chi(\|w\|)=$ $\mu\|w\|^{2} \in \mathcal{K}$ and $d=\epsilon_{0}^{2} / 4 c>0$. The conclusion follows by Proposition 1

Remark 11. The assumption $\dot{V} \leq-\alpha_{0} V+\mu\|w\|^{2}$ in Theorem 3 and 4 can be verified by using Theorem [1] Therefore, Theorem 1,3 and 4 altogether provide a systematic and constructive approach to design observer-based event-triggered controllers. One limitation of this ETC design, however, is that it relies on the global Lipschitz constant which is normally very conservatively computed.

Remark 12. Similar to Remark 10 there are trade-offs in choosing parameters in triggering rules (83) and (85); for example, smaller $\epsilon_{u}, \epsilon_{y}$ reduces the ultimate bounds but decreases the inter-execution times.

\section{Simulation Example}

In this section, we use a single-link robot arm example given in [62] and the configuration of Figure 2 to illustrate Theorem 4 Dynamics of the single-link robot arm are expressed as:

$$
\begin{aligned}
\dot{x}_{1} & =x_{2}, \\
\dot{x}_{2} & =-\sin \left(x_{1}\right)+u+w, \\
y & =x_{1},
\end{aligned}
$$

where $x=\left(x_{1}, x_{2}\right)^{\top}$ is the state representing the angle and the rotational velocity, $u$ is the input representing the torque, and $w$ is the external disturbance. The system can be written in the form of 11 with $A=\left(\begin{array}{ll}0 & 1 \\ 0 & 0\end{array}\right), B=\left(\begin{array}{l}0 \\ 1\end{array}\right)$, $C=(1,0), D=0, E=\left(\begin{array}{c}0 \\ -1\end{array}\right), E_{w}=\left(\begin{array}{l}0 \\ 1\end{array}\right), F_{w}=0$, $C_{q}=(1,0)$ and $p(q)=\sin (q)$. The nonlinearity $p$ satisfies (2) with $M=\left(\begin{array}{cc}1 & 0 \\ 0 & -1\end{array}\right)$. Recalling Remark 2 $p$ satisfies Assumption 11 and 2 with $T_{1}=T_{2}=\left(\begin{array}{ll}I & \mathbf{0} \\ \mathbf{0} & I\end{array}\right)$ and $\mathcal{N}_{1}=\left\{\left(\lambda_{1} I, \lambda_{1} I\right) \mid \lambda_{1}>0\right\}, \mathcal{N}_{2}=\left\{\left(\frac{1}{\lambda_{2}} I, \frac{1}{\lambda_{2}} I\right) \mid \lambda_{2}>0\right\}$, which means that $X_{1}=Y_{1}=\lambda_{1} I, X_{2} \stackrel{\lambda_{2}}{=} Y_{2}=\lambda_{2} I$. Additionally, the corresponding $M_{24}=-1<0$. By letting $\alpha_{1}=\alpha_{2}=1, \mu_{1}=\mu_{2}=0.1$, the LMIs 29)-30 with variables $\lambda_{1}, \lambda_{2}, P_{1}, P_{2}$ are feasible, from which we can obtain matrix gains $L_{1}=-1, L_{2}=\left(\begin{array}{c}-5.1294 \\ -18.0352\end{array}\right)$, $K_{1}=(-7.3936,-3.9937), K_{2}=1$. The observer is given in (84) with $L_{1}, L_{2}$ above, and the controller is given in 71 with $K_{1}, K_{2}$ above. We then let $\alpha_{0}=0.25, w_{0}=0.02$ and recompute $P$ via (20) with the objective to be minimizing the condition number of $P$. With $\varrho=0.8, a_{1}=a_{2}=0.5$, $\epsilon_{u}=\epsilon_{y}=0.005$, we can calculate that $\sigma_{y}=0.0017$, $\sigma_{u}=0.0023$, and $\tau_{u} \geq 1.07 \times 10^{-4} \mathrm{~s}, \tau_{u} \geq 7.68 \times 10^{-5}$ s. In the simulations, we suppose that the random disturbance $w$ is uniformly generated from $\left[-w_{0}, w_{0}\right]$, and the initial conditions of the plant and the observer are $(0.1,-0.15)$ and $(-0.1,0.05)$, respectively. The simulation results are shown in Figure 3 through Figure 7 .

Figure 3 and Figure 4 show trajectories of the state $x$ and the estimation error $e$, respectively. Both $x$ and $e$ eventually enter a small neighborhood of the origin as expected. Figure 5 shows inter-execution times $\left\{t_{k+1}^{y}-t_{k}^{y}\right\}$ in the observer ETM (83), and Figure 6 shows inter-execution times $\left\{t_{k+1}^{u}-t_{k}^{u}\right\}$ in the controller ETM (85). Figure 7 shows the trajectory of the piecewise constant input $u(t)$ that is fed into the plant. It is readily seen that the control input $u(t)$ updates its values at each sampling time $t=t_{k}^{u}$, which is determined by the triggering rule 85 . 
Denote $\tau_{\left[T_{1}, T_{2}\right]}^{\min }$ and $\tau_{\left[T_{1}, T_{2}\right]}^{a v g}$ as the minimal and average inter-execution times during the time interval $\left[T_{1}, T_{2}\right]$, respectively. The values of $\tau_{\text {min }}^{[0,20]}, \tau_{\text {avg }}^{[0,20]}, \tau_{\text {min }}^{[3,20]}, \tau_{\text {avg }}^{[3,20]}$ for the observer ETM and the controller ETM are summarized in Table I]. We notice that after 3 seconds, the controller input is updated about every 0.36 seconds on average, and the plant output is updated about every 1.09 seconds on average, which shows the effectiveness of our control design.

TABLE I

Minimal AND AVERAGE INTER-EXECUTION TIMES FOR OBSERVER AND CONTROLLER ETMS

\begin{tabular}{c|c|c|c|c}
\hline & $\tau_{[0,20]}^{\min }$ & $\tau_{[0,20]}^{a v g}$ & $\tau_{[3,20]}^{\min }$ & $\tau_{[3,20]}^{a v g}$ \\
\hline Observer ETM & $0.0106 \mathrm{~s}$ & $0.1945 \mathrm{~s}$ & $0.2104 \mathrm{~s}$ & $1.0977 \mathrm{~s}$ \\
\hline Controller ETM & $0.0013 \mathrm{~s}$ & $0.0663 \mathrm{~s}$ & $0.0903 \mathrm{~s}$ & $0.3665 \mathrm{~s}$ \\
\hline
\end{tabular}

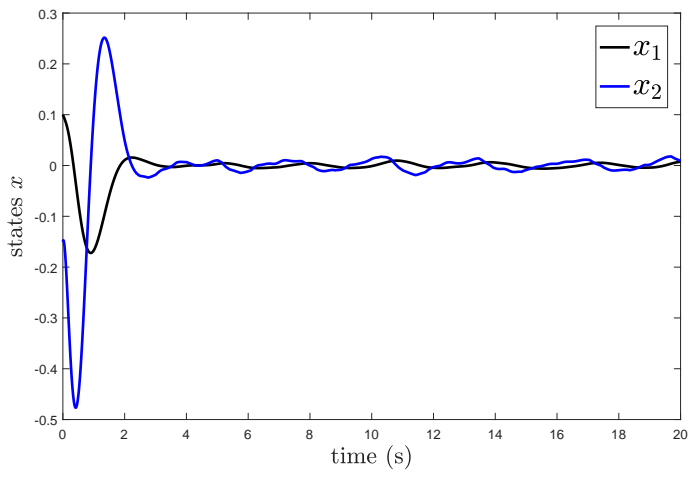

Fig. 3. Trajectory of the plant state $x$.

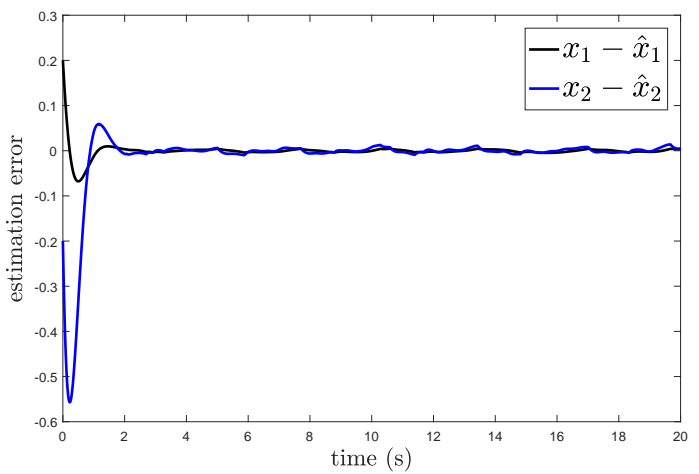

Fig. 4. Trajectory of the estimation error $e=x-\hat{x}$.

\section{CONCLUSION}

In this paper, we studied observer-based, global stabilizing control design for incrementally quadratic nonlinear systems affected by external disturbances and measurement noise. We proposed LMI-based sufficient conditions for the simultaneous design of the observer and the controller in the continuoustime domain for two parameterizations of the incremental multiplier matrices. Based on that, we investigated ETM design within the observer-based controller setting for globally

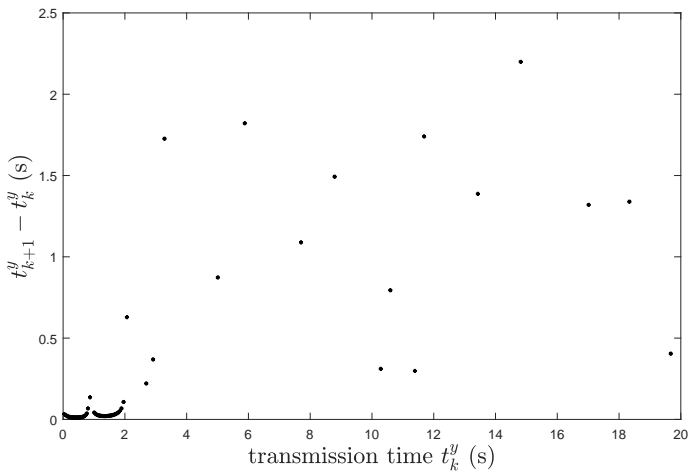

Fig. 5. Inter-execution times $\left\{t_{k+1}^{y}-t_{k}^{y}\right\}$ in the observer ETM 83 .

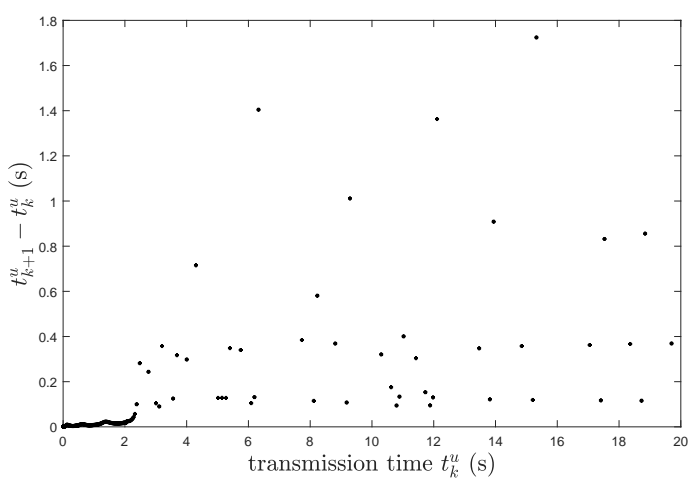

Fig. 6. Inter-execution times $\left\{t_{k+1}^{u}-t_{k}^{u}\right\}$ in the controller ETM 85.

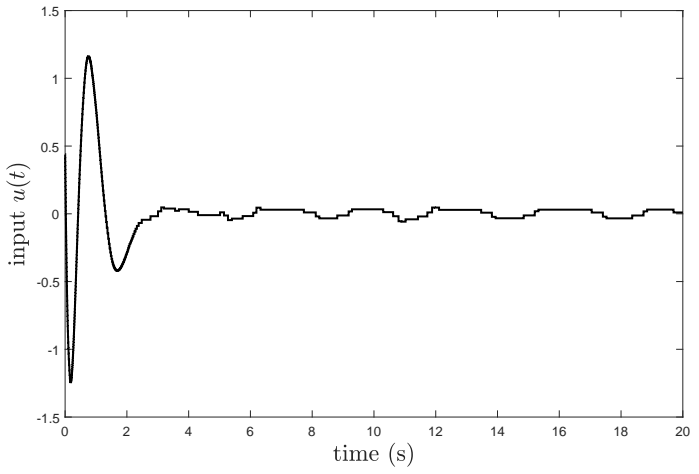

Fig. 7. Trajectory of the input $u(t)$.

Lipschitz systems. The simulation example showed the effectiveness of the controller design and the proposed triggering rule.

\section{REFERENCES}

[1] A. J. Krener and A. Isidori, "Linearization by output injection and nonlinear observers," Systems \& Control Letters, vol. 3, no. 1, pp. 47-52, 1983.

[2] M. Vidyasagar, "On the stabilization of nonlinear systems using state detection," IEEE Transactions on Automatic Control, vol. 25, no. 3, pp. 504-509, 1980.

[3] M. Krstic, I. Kanellakopoulos, and P. V. Kokotovic, Nonlinear and adaptive control design. Wiley, 1995.

[4] V. Andrieu and L. Praly, "A unifying point of view on output feedback designs for global asymptotic stabilization," Automatica, vol. 45 , no. 8 , pp. 1789-1798, 2009. 
[5] A. Borri, F. Cacace, A. De Gaetano, A. Germani, C. Manes, P. Palumbo, S. Panunzi, and P. Pepe, "Luenberger-like observers for nonlinear timedelay systems with application to the artificial pancreas: The attainment of good performance," IEEE Control Systems Magazine, vol. 37, no. 4, pp. 33-49, 2017.

[6] C. C. Cheah, X. Li, X. Yan, and D. Sun, "Observer-based optical manipulation of biological cells with robotic tweezers," IEEE Transactions on Robotics, vol. 30, no. 1, pp. 68-80, 2014.

[7] H. O. Méndez-Acosta, R. Femat, and V. González-Álvarez, Selected topics in dynamics and control of chemical and biological processes. Springer, 2007, vol. 361.

[8] H. K. Khalil, "High-gain observers in feedback control: Application to permanent magnet synchronous motors," IEEE Control Systems Magazine, vol. 37, no. 3, pp. 25-41, 2017.

[9] R. Rajamani, Y. Wang, G. D. Nelson, R. Madson, and A. Zemouche, "Observers with dual spatially separated sensors for enhanced estimation: Industrial, automotive, and biomedical applications," IEEE Control Systems Magazine, vol. 37, no. 3, pp. 42-58, 2017.

[10] Y. Su, P. C. Muller, and C. Zheng, "A simple nonlinear observer for a class of uncertain mechanical systems," IEEE Transactions on Automatic Control, vol. 52, no. 7, pp. 1340-1345, 2007.

[11] M. Ouassaid, M. Maaroufi, and M. Cherkaoui, "Observer-based nonlinear control of power system using sliding mode control strategy," Electric Power Systems Research, vol. 84, no. 1, pp. 135-143, 2012.

[12] M. A. Mahmud, H. Pota, and M. Hossain, "Full-order nonlinear observer-based excitation controller design for interconnected power systems via exact linearization approach," International Journal of Electrical Power \& Energy Systems, vol. 41, no. 1, pp. 54-62, 2012.

[13] Y. Hong, G. Chen, and L. Bushnell, "Distributed observers design for leader-following control of multi-agent networks," Automatica, vol. 44, no. 3, pp. 846-850, 2008.

[14] T. Ahmed-Ali and F. Lamnabhi-Lagarrigue, "High gain observer design for some networked control systems," IEEE Transactions on Automatic Control, vol. 57, no. 4, pp. 995-1000, 2012.

[15] P. V. Kokotovic, "The joy of feedback: nonlinear and adaptive," IEEE Control Systems Magazine, vol. 12, no. 3, pp. 7-17, 1992

[16] F. Mazenc, L. Praly, and W. Dayawansa, "Global stabilization by output feedback: examples and counterexamples," Systems \& Control Letters, vol. 23, no. 2, pp. 119-125, 1994.

[17] P. Kokotović and M. Arcak, "Constructive nonlinear control: a historical perspective," Automatica, vol. 37, no. 5, pp. 637-662, 2001.

[18] F. Esfandiari and H. K. Khalil, "Output feedback stabilization of fully linearizable systems," International Journal of control, vol. 56, no. 5, pp. 1007-1037, 1992

[19] H. K. Khalil and L. Praly, "High-gain observers in nonlinear feedback control," International Journal of Robust and Nonlinear Control, vol. 24, no. 6, pp. 993-1015, 2014.

[20] A. Teel and L. Praly, "Global stabilizability and observability imply semi-global stabilizability by output feedback," Systems \& Control Letters, vol. 22, no. 5, pp. 313-325, 1994

[21] H. Khalil and F. Esfandiari, "Semiglobal stabilization of a class of nonlinear systems using output feedback," IEEE Transactions on Automatic Control, vol. 38, no. 9, pp. 1412-1415, 1993.

[22] A. Atassi and H. Khalil, "Separation results for the stabilization of nonlinear systems using different high-gain observer designs," Systems \& Control Letters, vol. 39, no. 3, pp. 183-191, 2000.

[23] _ - "A separation principle for the control of a class of nonlinear systems," IEEE Transactions on Automatic Control, vol. 46, no. 5, pp. 742-746, 2001

[24] J. Gauthier and I. Kupka, "A separation principle for bilinear systems with dissipative drift," IEEE transactions on automatic control, vol. 37, no. 12, pp. 1970-1974, 1992

[25] J. Tsinias, "Sontag's input to state stability conditionand global stabilization using state detection," Systems \& Control Letters, vol. 20, no. 3, pp. 219-226, 1993.

[26] W. Lin, "Bounded smooth state feedback and a global separation principle for non-affine nonlinear systems," Systems \& Control Letters, vol. 26, no. 1, pp. 41-53, 1995.

[27] M. Arcak and P. Kokotović, "Nonlinear observers: a circle criterion design and robustness analysis," Automatica, vol. 37, no. 12, pp. 19231930, 2001.

[28] M. Arcak and P. Kokotovic, "Observer-based control of systems with slope-restricted nonlinearities," IEEE Transactions on Automatic Control, vol. 46, no. 7, pp. 1146-1150, 2001.

[29] M. Arcak, "Certainty-equivalence output-feedback design with circlecriterion observers," IEEE Transactions on Automatic Control, vol. 50, no. 6, pp. 905-909, 2005
[30] T. I. Fossen and J. P. Strand, "Passive nonlinear observer design for ships using lyapunov methods: full-scale experiments with a supply vessel," Automatica, vol. 35, no. 1, pp. 3-16, 1999.

[31] A. Loria, T. I. Fossen, and E. Panteley, "A separation principle for dynamic positioning of ships: Theoretical and experimental results," IEEE Transactions on Control Systems Technology, vol. 8, no. 2, pp. 332-343, 2000

[32] L. Praly and Z.-P. Jiang, "Stabilization by output feedback for systems with ISS inverse dynamics," Systems \& Control Letters, vol. 21, no. 1, pp. 19-33, 1993.

[33] J.-B. Pomet, R. M. Hirschorn, and W. Cebuhar, "Dynamic output feedback regulation for a class of nonlinear systems," Mathematics of Control, Signals and Systems, vol. 6, no. 2, pp. 106-124, 1993.

[34] L. Praly, "Lyapunov design of a dynamic output feedback for systems linear in their unmeasured state components," in Nonlinear Control Systems Design. Pergamon, 1993, pp. 63-68.

[35] S. Boyd, L. El Ghaoui, E. Feron, and V. Balakrishnan, Linear matrix inequalities in system and control theory. SIAM, 1994.

[36] C.-H. Lien, "Robust observer-based control of systems with state perturbations via LMI approach," IEEE Transactions on Automatic Control, vol. 49, no. 8, pp. 1365-1370, 2004.

[37] H. Kheloufi, A. Zemouche, F. Bedouhene, and M. Boutayeb, "On LMI conditions to design observer-based controllers for linear systems with parameter uncertainties," Automatica, vol. 49, no. 12, pp. 3700-3704, 2013.

[38] A. Zemouche, R. Rajamani, H. Kheloufi, and F. Bedouhene, "Robust observer-based stabilization of Lipschitz nonlinear uncertain systems via LMIs-discussions and new design procedure," International Journal of Robust and Nonlinear Control, vol. 27, no. 11, pp. 1915-1939, 2017.

[39] O. Toker and H. Ozbay, "On the NP-hardness of solving bilinear matrix inequalities and simultaneous stabilization with static output feedback," in American Control Conference. IEEE, 1995, pp. 2525-2526.

[40] Y. Wang, R. Rajamani, and A. Zemouche, "Sequential LMI approach for the design of a BMI-based robust observer state feedback controller with nonlinear uncertainties," International Journal of Robust and Nonlinear Control, vol. 28, no. 4, pp. 1246-1260, 2018.

[41] K.-K. K. Kim and R. D. Braatz, "Robust static and fixed-order dynamic output feedback control of discrete-time parametric uncertain luré systems: Sequential sdp relaxation approaches," Optimal Control Applications and Methods, vol. 38, no. 1, pp. 36-58, 2017.

[42] B. Grandvallet, A. Zemouche, H. Souley-Ali, and M. Boutayeb, "New LMI condition for observer-based $\mathrm{H}_{\infty}$ stabilization of a class of nonlinear discrete-time systems," SIAM Journal on Control and Optimization, vol. 51, no. 1, pp. 784-800, 2013.

[43] M. Ekramian, "Observer-based controller for Lipschitz nonlinear systems," International Journal of Systems Science, vol. 48, no. 16, pp. 3411-3418, 2017.

[44] A. Megretski and A. Rantzer, "System analysis via integral quadratic constraints," IEEE Transactions on Automatic Control, vol. 42, no. 6 , pp. 819-830, 1997.

[45] B. Açıkmeşe, "Stabilization, observation, tracking and disturbance rejection for uncertain/nonlinear and time-varying systems," Ph.D. dissertation, Purdue University, 2002.

[46] B. Açıkmeşe and M. Corless, "Observers for systems with nonlinearities satisfying an incremental quadratic inequality," in American Control Conference, 2005, pp. 3622-3629.

[47] _ _ "Stability analysis with quadratic lyapunov functions: some necessary and sufficient multiplier conditions," Systems \& Control Letters, vol. 57, no. 1, pp. 78-94, 2008.

[48] — - "Observers for systems with nonlinearities satisfying incremental quadratic constraints," Automatica, vol. 47, no. 7, pp. 1339-1348, 2011.

[49] L. D'Alto and M. Corless, "Incremental quadratic stability," Numerical Algebra, Control and Optimization, vol. 3, no. 1, pp. 175-201, 2013.

[50] A. Chakrabarty, M. Corless, G. T. Buzzard, S. H. Zak, and A. E. Rundell, "State and unknown input observers for nonlinear systems with bounded exogenous inputs," IEEE Transactions on Automatic Control, vol. 62, no. 11 , pp. 5497-5510, 2017.

[51] M.-S. Chen and C.-C. Chen, "Robust nonlinear observer for Lipschitz nonlinear systems subject to disturbances," IEEE Transactions on Automatic control, vol. 52, no. 12, pp. 2365-2369, 2007.

[52] M. Arcak and P. Kokotović, "Observer-based stabilization of systems with monotonic nonlinearities," Asian Journal of Control, vol. 1, no. 1 , pp. 42-48, 1999.

[53] X. Fan and M. Arcak, "Observer design for systems with multivariable monotone nonlinearities," Systems \& Control Letters, vol. 50, no. 4, pp. 319-330, 2003 
[54] W. Heemels, K. H. Johansson, and P. Tabuada, "An introduction to eventtriggered and self-triggered control," in IEEE Conference on Decision and Control, 2012, pp. 3270-3285.

[55] P. Tabuada, "Event-triggered real-time scheduling of stabilizing control tasks," IEEE Transactions on Automatic Control, vol. 52, no. 9, pp. 1680-1685, 2007.

[56] R. Postoyan, P. Tabuada, D. Nešić, and A. Anta, "A framework for the event-triggered stabilization of nonlinear systems," IEEE Transactions on Automatic Control, vol. 60, no. 4, pp. 982-996, 2015.

[57] P. Tallapragada and N. Chopra, "Event-triggered dynamic output feedback control for LTI systems," in IEEE Conference on Decision and Control, 2012, pp. 6597-6602.

[58] S. Tarbouriech, A. Seuret, J. M. G. da Silva Jr, and D. Sbarbaro, "Observer-based event-triggered control co-design for linear systems," IET Control Theory \& Applications, vol. 10, no. 18, pp. 2466-2473, 2016.

[59] M. Donkers and W. Heemels, "Output-based event-triggered control with guaranteed $\mathcal{L}_{\infty}$-gain and improved and decentralized event-triggering," IEEE Transactions on Automatic Control, vol. 57, no. 6, pp. 1362-1376, 2012.

[60] D. N. Borgers and W. M. Heemels, "Event-separation properties of event-triggered control systems," IEEE Transactions on Automatic Control, vol. 59, no. 10, pp. 2644-2656, 2014.

[61] V. Dolk, D. P. Borgers, and W. Heemels, "Output-based and decentralized dynamic event-triggered control with guaranteed $\mathcal{L}_{p}$-gain performance and zeno-freeness," IEEE Transactions on Automatic Control, vol. 62, no. 1, pp. 34-49, 2017.

[62] M. Abdelrahim, R. Postoyan, J. Daafouz, and D. Nešić, "Robust eventtriggered output feedback controllers for nonlinear systems," Automatica, vol. 75, pp. 96-108, 2017.

[63] X. Xu, B. Açıkmeşe, M. Corless, and H. Sartipizadeh, "Observer-based output feedback control design for systems with incrementally conic nonlinearities," in American Control Conference, 2018, pp. 1364-1369.

[64] Z.-P. Jiang, I. M. Mareels, and Y. Wang, "A Lyapunov formulation of the nonlinear small-gain theorem for interconnected ISS systems," Automatica, vol. 32, no. 8, pp. 1211-1215, 1996.

[65] A. Isidori, Nonlinear Control Systems-II. Springer Science \& Business Media, 2013.

[66] E. D. Sontag and Y. Wang, "On characterizations of the input-to-state stability property," Systems \& Control Letters, vol. 24, no. 5, pp. 351359, 1995. 